\title{
Sila-biperiden und endo-Sila-biperiden: Synthesen, Kristallstrukturen und antimuscarinische Eigenschaften
}

\author{
Reinhold Tacke, Jerzy Pikies und Frank Wiesenberger \\ Institut für Anorganische Chemie, Universität Karlsnuhe, Engesserstraße, Geb. 30.45, D-76128 Karlsruhe (Deutschland)
}

\section{Ludger Ernst}

NMR-Laboratorium der Chemischen Institute, Technische Universität Braunschweig, Hagenring 30, D-38106 Braunschweig (Deutschland)

\author{
Dietmar Schomburg \\ Gesellschaft für Biotechnologische Forschung (GBF), Mascheroder Weg 1, D-38124 Braunschweig (Deutschland)
}

\section{Magali Waelbroeck und Jean Christophe}

Laboratoire de Chimie Biologique et de la Nutrition, Faculté de Médecine et de Pharmacie, Université Libre de Bruxelles, Route de Lennik 808, B-1070 Bruxelles (Belgique)

\section{Günter Lambrecht, Jan Gross und Ernst Mutschler}

Pharmakologisches Institut für Naturwissenschafiler, Universität Frankfurt, Biozentrum Niederursel, Marie-Curie-Straße 9, Geb. N260, D-60053 Frankfurt (Deutschland)

(Eingegangen den 10. Mai 1993)

\begin{abstract}
Starting from trichloro(vinyl)silane $\left(\mathrm{Cl}_{3} \mathrm{SiCH}=\mathrm{CH}_{2}\right)$, the muscarinic antagonists sila-biperiden [ $\mathrm{rac}-(\mathrm{Si} R S, \mathrm{C} 2 \mathrm{SR}$ )exo-2] and endosila-biperiden [ rac-(SiRS,C2SR)-endo-2] were prepared by a seven-step synthesis. Both silanols are configurationally stable in inert organic solvents but undergo slow epimerization in aqueous solution $\left(\mathrm{pH} 7.4,32^{\circ} \mathrm{C}\right)$ by inversion of the configuration at the silicon atom. The relative configurations of sila-biperiden and endo-sila-biperiden were determined by single-crystal X-ray diffraction. Both compounds form intermolecular $\mathrm{O}-\mathrm{H} \cdots \mathrm{N}$ hydrogen bonds in the crystal leading to the formation of centrosymmetric dimers (sila-biperiden) and infinite chains (endo-sila-biperiden), respectively. Sila-biperiden is a silicon analogue (C/Si exchange) of the antiparkinsonian drug biperiden [ rac-(CRS/C2SR)-exo-1]. In functional pharmacological experiments, as well as in radioligand competition studies, biperiden, sila-biperiden and endo-sila-biperiden behaved as simple competitive antagonists at muscarinic M1-, M2-, M3- and M4-receptors. The three compounds displayed the highest affinity for M1-receptors (pA $\mathrm{A}_{2}$ values: 8.72-8.80; $\mathrm{pK}_{\mathrm{i}}$ values: 8.8-9.1), intermediate affinity for $\mathrm{M4}$ - and $\mathrm{M3}$-receptors, and lowest affinity for $\mathrm{M} 2$-receptors ( $\mathrm{pA}_{2}$ values: 7.57-7.79; $\mathrm{pK}_{\mathrm{i}}$ values: 7.7-7.8). The affinity profile $(\mathrm{M} 1>\mathrm{M} 4>\mathrm{M} 3>\mathrm{M} 2)$ of biperiden, sila-biperiden and endo-sila-biperiden is qualitatively similar to that of the M1-selective muscarinic antagonist pirenzepine. The antimuscarinic properties of the $\mathrm{C} / \mathrm{Si}$ analogues biperiden and sila-biperiden are almost identical.
\end{abstract}

\section{Zusammenfassung}

Die Antimuscarinica Sila-biperiden [ rac-(Si $R S, C 2 S R$ )exo-2] und endo-Sila-biperiden [ rac-(Si RS,C2SR)-endo-2] wurden ausgehend von Trichlor(vinyl)silan $\left(\mathrm{Cl}_{3} \mathrm{SiCH}=\mathrm{CH}_{2}\right)$ durch eine siebenstufige Synthese dargestellt. Die beiden Silanole sind in inerten organischen Solvenzien konfigurationsstabil, unterliegen aber in wässeriger Lösung ( $\mathrm{pH} 7.4,32^{\circ} \mathrm{C}$ ) einer Epimerisierung durch Inversion der Konfiguration am Silicium-Atom. Die relativen Konfigurationen von Sila-biperiden und endo-Sila-biperiden wurden 
durch Einkristall-Röntgenstrukturanalysen bestimmt. Beide Verbindungen bilden im Kristall intermolekulare O-H $\cdots \mathrm{N}$-Wasserstoff-Brückenbindungen aus, die zum Aufbau von zentrosymmetrischen Dimeren (Sila-biperiden) bzw. unendlichen Ketten (endo-Sila-biperiden) führen. Sila-biperiden ist ein Silicium-Analogon (C/Si-Austausch) des Antiparkinsonmittels Biperiden [ rac-(CRS,C2SR)-exo-1]. Sowohl in funktionellen pharmakologischen Untersuchungen als auch in Radioligand-Kompetitionsexperimenten erwiesen sich Biperiden, Sila-biperiden und endo-Sila-biperiden als rein kompetitive Antagonisten an muscarinischen M1-, M2-, M3- und M4-Rezeptoren. Alle drei Verbindungen zeigten die höchste Affinität zu den M1-Rezeptoren ( $\mathrm{pA}_{2}$-Werte: 8.72-8.80; $\mathrm{pK}_{\mathrm{i}}$-Werte: 8.8-9.1), eine deutlich geringere Affinität zu den M4- und M3-Rezeptoren und die niedrigste Affinität zu den kardialen M2-Rezeptoren ( $\mathrm{pA}_{2}$-Werte: 7.57-7.79; $\mathrm{pK}_{i}$-Werte: 7.7-7.8). Das Affinitätsprofil (M1 $>\mathrm{M} 4>\mathrm{M} 3>\mathrm{M} 2$ ) von Biperiden, Sila-biperiden und endo-Sila-biperiden ist dem des M1-selektiven Antimuscarinicums Pirenzepin qualitativ sehr ähnlich. Die antimuscarinischen Eigenschaften der C/Si-Analoga Biperiden und Sila-biperiden sind nahezu identisch.

Key words: Silicon; Silanol; Sila-biperiden; Bioorganosilicon chemistry; Muscarinic antagonist; Muscarinic receptor subtype

\section{Einleitung}

In Fortführung unserer systematischen Untersuchungen über sila-substituierte Pharmaka vom Typ des Sila-pridinols und Sila-difenidols (ausgewähite Arbeiten: [1-16]) haben wir das racemische Sila-biperiden [ rac-(Si RS,C2SR)-exo-2] und endo-Sila-biperiden [ rac-(Si $R S, C 2 S R)$-endo-2] synthetisiert und pharmakologisch charakterisiert. Sila-biperiden ist ein Silicium-Analogon des Antimuscarinicums Biperiden [ rac(CRS,C2SR)-exo-1], das zur Therapie der Parkinsonschen. Krankheit eingesetzt wird (Handelspräparat:

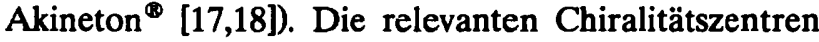
[Si (bzw. C) und C2] der genannten Verbindungen sind in den nachstehenden Formeln jeweils mit einem Stern gekennzeichnet.

Wir berichten hier über die Synthesen, Kristallstrukturanalysen und antimuscarinischen Eigenschaften der Silicium-Verbindungen Sila-biperiden und endo-Silabiperiden. Die Bestimmung der antimuscarinischen Eigenschaften, in die auch das Kohlenstoff-Analogon Biperiden einbezogen wurde, erfolgte sowohl durch funktionelle pharmakologische Untersuchungen an muscarinischen M1-, M2- und M3-Rezeptoren als auch
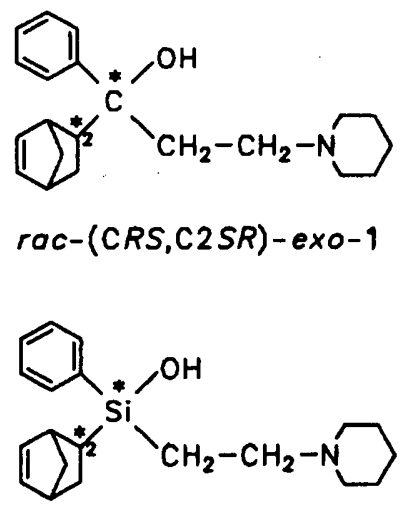

rac-(SiRS,C2SR)-exo-2 rac-(SiRS,C2SR)-endo-2

Schema 1. durch Radioligand-Bindungsstudien an M1-, M2-, M3und M4-Rezeptoren.

\section{Ergebnisse und Diskussion}

\subsection{Synthesen}

Sila-biperiden [ rac-(Si $R S, C 2 S R)$ exo-2] und endoSila-biperiden [ $\mathrm{rac}$-(Si $R S, \mathrm{C} 2 S R$ )-endo-2] wurden ausgehend von Trichlor(vinyl)silan (3) gemäß Schema 2 durch eine siebenstufige Synthese hergestellt. Die beiden racemischen Silanole fielen hierbei zusammen mit den diastereomeren Racematen rac-(Si $R S, \mathrm{C} 2 R S)$-exo2 und rac-(Si $R S, C 2 R S)$-endo-2 als ein Gemisch von Feststoffen an, aus dem Sila-biperiden und endo-Silabiperiden durch fraktionierende Kristallisation rein isoliert werden konnten.

Durch Umsetzung von 3 mit Cyclopentadien wurde zunächst nach bekanntem Verfahren [19] ein Gemisch aus rac-(endo-Bicyclo[2.2.1]hept-5-en-2-yl)trichlorsilan [ rac-(C2RS)-endo-4] und rac-(exo-Bicyclo[2.2.1]hept-5en-2-yl)trichlorsilan [ rac-(C2RS)-exo-4] hergestellt (Ausbeute 89\%, endo/exo-Verhältnis 7:3). Durch Methanolyse dieses Trichlorsilan-Gemisches in Gegenwart von Triethylamin erhielt man ein Gemisch aus den entsprechenden Trimethoxysilanen rac-(C2RS). endo-5 und rac-(C2RS)-exo-5 (Ausbeute $84 \%$, endo / exo-Verhältnis 7:3). Die nachfolgende Umsetzung mit Phenylmagnesiumbromid führte dann $\mathrm{zu}$ einem Gemisch der Phenylsilane rac-(C2RS) endo-6 und rac(C2RS)-exo-6 (Ausbeute 92\%, endo / exo-Verhältnis 7:3). Die Umsetzung dieses Dimethoxysilan-Gemisches mit einem Äquivalent Vinylmagnesiumchlorid und anschließend mit Lithiumaluminiumhydrid ergab dann unter Aufbau eines weiteren Asymmetriezentrums (Silicium-Atom) ein Gemisch aus den entsprechenden Vinylhydridosilanen rac-(Si $R S, \mathrm{C} 2 R S)$-endo-7, rac(Si $R S, C 2 S R$ )-ndo-7, rac-(Si $R S, C 2 R S$ )-exo-7 und rac(Si RS,C2SR)-exo-7 (Ausbeute 52\%, Mol-Verhältnis 7:7:3:3). Dieses Vinylhydridosilan-Gemisch wurde dann mit Piperidin und dessen Lithiumamid umgesetzt, wobei man unter Substitution der SiH-Wasserstoff-Atome durch den Piperidino-Rest und unter Ad- 
dition von Piperidin an die Vinyl-Gruppen ein Gemisch aus den entsprechenden Piperidino(2-piperidinoethyl)silanen rac-(Si $R S, \mathrm{C} 2 R S$ )-endo-8, rac-(Si $R S$, $\mathrm{C} 2 S R$ )-endo-8, rac-(SiRS,C2RS)-exo-8 und rac(Si RS,C2SR)-exo-8 erhieit (Ausbeute 82\%, MolVerhältnis 7:7:3:3). Die Methanolyse dieses Piperidinosilan-Gemisches führte zu einem Gemisch der Methoxysilane rac-(Si $R S, \mathrm{C} 2 R S)$-endo-9, rac-(Si $R S$, C2SR)-endo-9, rac-(Si $R S, \mathrm{C} 2 R S)$-exo-9 und rac-(Si $R S$, C2SR)-exo-9 (Ausbeute 94\%, Mol-Verhältnis
7:7:3:3). Die nachfolgende Hydrolyse ergab dann schließlich ein Gemisch aus den entsprechenden Silanolen rac-(Si $R S, \mathrm{C} 2 R S$ )-endo-2, rac-(Si $R S, \mathrm{C} 2 S R)$ endo-2) (endo-Sila-biperiden), rac-(Si $R S, C 2 R S$ )-exo-2 und rac-(SiRS,C2SR)-exo-2 (Sila-biperiden) (Ausbeute 93\%, Mol-Verhältnis 7:7:3:3).

Während die Silane 4-9 allesamt als Flüssigkeiten anfielen, die sich nicht in die jeweiligen Isomere auftrennen ließen, wurden die Silanole 2 als ein Gemisch von Feststoffen erhalten, aus dem man durch<smiles>C=C[Si](Cl)(Cl)Cl</smiles>

3

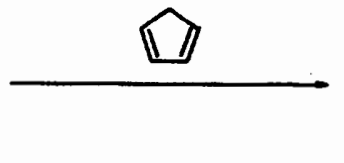

$\mathrm{C}_{8} \mathrm{H}_{5} \mathrm{MgBr}$<smiles>CO[Si](OC)(c1ccccc1)c1ccccc1</smiles>

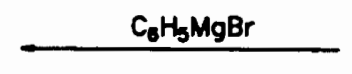

0-6

1. $\mathrm{CH}_{2}-\mathrm{CHMgCl}$

2. $\mathrm{LiAlH}_{4}$<smiles>COc1ccccc1[Si](Cl)(Cl)Cl</smiles><smiles>CO[Si](OC)(OC)c1ccccc1</smiles><smiles>CCC[Si](c1ccccc1)(c1ccccc1)c1ccccc1</smiles>

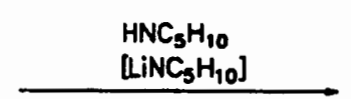

$\mathrm{H}_{2} \mathrm{O}$<smiles>CCCN1CCCCC1</smiles><smiles>[10BH]</smiles>

$\mathrm{CH}_{3} \mathrm{OH}$<smiles>CO[Si](C)(CCCN1CCCCC1)c1ccccc1</smiles>

o: rac-(C2RS)-endo, rac-(C2RS)-exo

b: rac-(SiRS,C2RS)-endo, rac-(SiRS,C2SR)-endo, rOC-(SiRS,C2RS)-exo, roc-(SiRS,C2SR)-exo

Schema 2. 
fraktionierende Kristallisation die beiden Verbindungen rac-(SiRS,C2SR)-exo-2 (Sila-biperiden) und rac(Si $R S, \mathrm{C} 2 S R$ )-endo-2 (endo-Sila-biperiden) abtrennen und jeweils rein isolieren konnte.

Die Konstitution der Silanole 2 wurde durch NMRspektroskopische und massenspektrometrische Untersuchungen sichergestellt; ihre Konfiguration wurde durch Einkristall-Röntgenstrukturanalysen an Sila-biperiden und endo-Sila-biperiden bestimmt [siehe Abschnitt 2.2.]. Die Identität der bisher noch nicht beschriebenen Silane 5-9 (Isomerengemische) wurde durch NMR-spektroskopische und massenspektrometrische Untersuchungen sowie durch Elementaranalysen sichergestellt.

Studien zur Konfigurationsstabilität von Sila-biperiden und endo-Sila-biperiden haben gezeigt, daß beide Silanole in unpolaren organischen Solvenzien beständig sind, in wässeriger Lösung jedoch durch Inversion der Konfiguration am Silicium-Atom epimerisieren [ $\rightarrow$ Bildung von rac-(Si $R S, C 2 R S)$-exo-2 bzw. rac-(Si $R S$, $\mathrm{C} 2 R S$ )-endo-2]. Orientierende Experimente ergaben, daß Sila-biperiden und endo-Sila-biperiden in wässeriger Lösung (Phosphat-Puffer, pH 7.4) bei $32^{\circ} \mathrm{C}$ in 15 Minuten zu etwa $10 \%$ epimerisieren. Diese Epimerisierung entspricht formal der Racemisierung, die wir bereits früher für die Enantiomere von Sila-procyclidin (10) und Sila-tricyclamoliodid (11) (Schema 3) in wässeriger Lösung beobachtet haben [9].

\subsection{Kristallstrukturanalysen}

Geeignete Einkristalle von Sila-biperiden [ rac(SiRS,C2SR)-exo-2] und endo-Sila-biperiden [rac( $\mathrm{Si} R S, \mathrm{C} 2 S R$ )-endo-2] wurden durch Kristallisation aus Diethylether erhalten. Die Ergebnisse der Kristallstrukturanalysen sind in den Abb. 1-4 und in den Tabellen 1-4 zusammengefaßt.

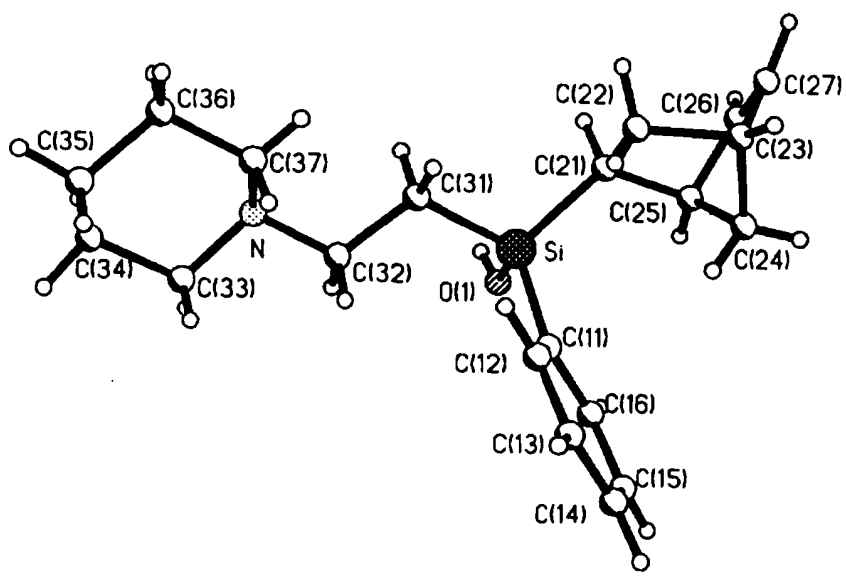

Abb. 1. Molekülstruktur des (SiR,C2S)-Enantiomers im Kristall von Sila-biperiden [rac-(SiRS,C2SR)-xo-2] mit Angabe des Numerierungsschemas.

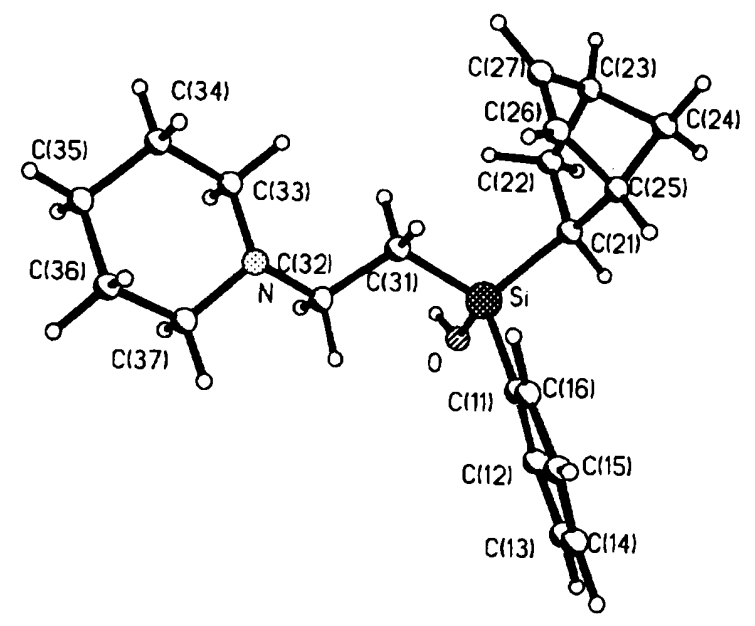

Abb. 2. Molekülstruktur des (Si $R, \mathrm{C} 2 S$ )-Enantiomers im Kristall von endo-Sila-biperiden [rac-(SiRS,C2SR)endo-2] mit Angabe des Numerierungsschemas.

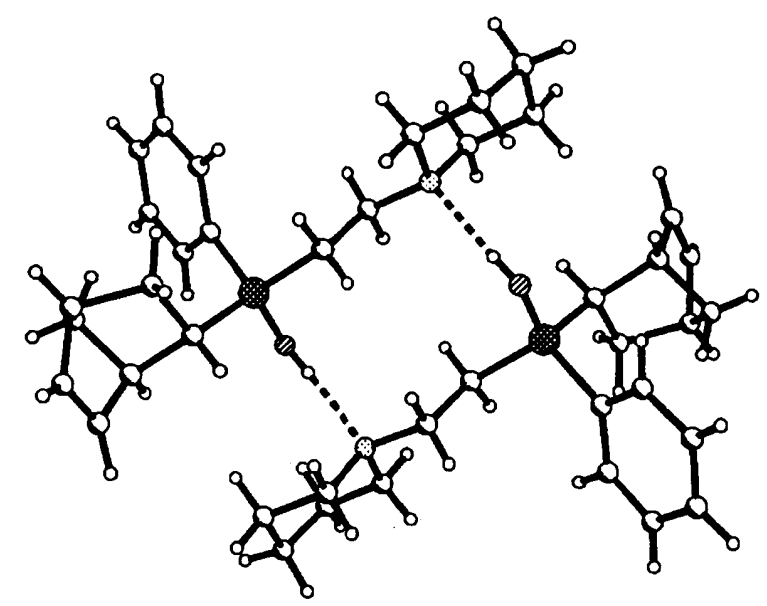

Abb. 3. Intermolekulare $\mathrm{O}-\mathrm{H} \cdots \mathrm{N}$-Wasserstoff-Brückenbindungen im Kristall von Sila-biperiden [ $\mathrm{rac}-(\mathrm{Si} R S, \mathrm{C} 2 S R)$-exo-2].

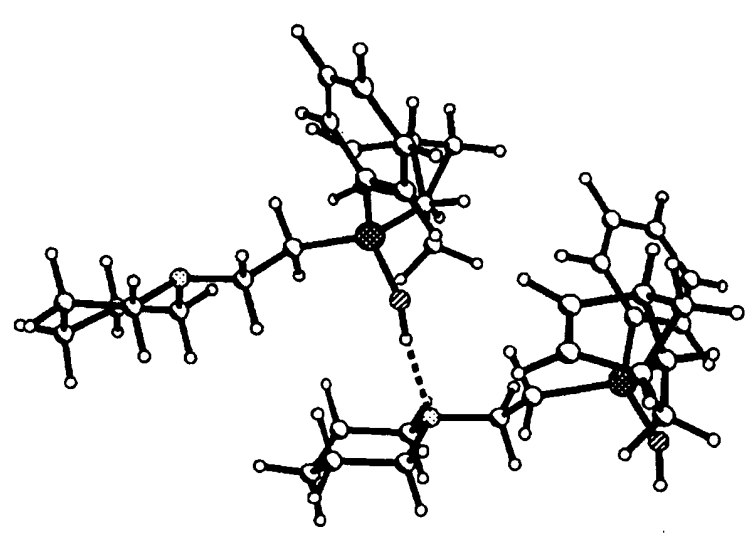

Abb. 4. Intermolekulare $\mathrm{O}-\mathrm{H} \cdots \mathrm{N}$-Wasserstoff-Brückenbindungen im Kristall von endo-Sila-biperiden [ rac-(Si $R S, C 2 S R$ )-endo-2]. 
<smiles>O[Si](CCN1CCCC1)(c1ccccc1)C1CCCCC1</smiles><smiles>C[N+]1(C)CCCC1</smiles>

Schema 3.

Die Molekülgeometrien von Sila-biperiden und endo-Sila-biperiden weisen eine Reihe auffälliger Gemeinsamkeiten auf (Abb. 1 und 2). Die beobachteten Si-O- [162.6(1) bzw. 162.1(3) pm] und Si-C-Abstände [186.3(4)-187.9(2) pm] liegen im Erwartungsbereich. Dies gilt auch - abgesehen von den relativ langen C(21)-C(25)-Abständen [157.4(2) bzw. 156.8(5) $\mathrm{pm}$ ] in den Bicyclo[2.2.1]hept-5-en-2-yl-Gruppen - für die $\mathrm{C}-\mathrm{C}$ - und $\mathrm{C}-\mathrm{N}$-Abstände beider Verbindungen. Auch die beobachteten Bindungswinkel zeigen keine Besonderheiten. Bezüglich der $C(31)-C(32)$-Bindung wurde in beiden Fällen eine anti-Konformation gefun-

TABELLE 1. Ortskoordinaten und atomare Auslenkungsparameter $\left(\mathrm{pm}^{2}\right)$ der Nichtwasserstoff-Atome von Sila-biperiden [rac(SiRS,C2SR)-exo-2]

\begin{tabular}{|c|c|c|c|c|}
\hline Atom & $x$ & $y$ & $z$ & $U_{e q}$ \\
\hline Si & $0.1401(0)$ & $0.4313(0)$ & $0.3496(0)$ & $0.037(0)$ \\
\hline O & $-0.0194(1)$ & $0.4425(1)$ & $0.2778(1)$ & $0.047(0)$ \\
\hline$C(11)$ & $0.2224(1)$ & $0.4861(1)$ & $0.2567(1)$ & $0.040(0)$ \\
\hline$C(12)$ & $0.3509(1)$ & $0.5208(1)$ & $0.3058(1)$ & $0.043(0)$ \\
\hline C(13) & $0.4139(1)$ & $0.5556(1)$ & $0.2348(2)$ & $0.050 \times 0)$ \\
\hline C(14) & $0.3506(2)$ & $0.5571(1)$ & $0.1137(2)$ & $0.058(0)$ \\
\hline$C(15)$ & $0.2233(2)$ & $0.5231(1)$ & $0.0618(1)$ & $0.063(0)$ \\
\hline C(16) & $0.1603(2)$ & $0.4883(1)$ & $0.1329(1)$ & $0.052(0)$ \\
\hline$C(21)$ & $0.1897(1)$ & $0.3142(1)$ & $0.3715(1)$ & $0.042(0)$ \\
\hline$C(22)$ & $0.3417(2)$ & $0.2964(1)$ & $0.4339(2)$ & $0.052(0)$ \\
\hline$C(23)$ & $0.3711(2)$ & $0.2285(1)$ & $0.3525(2)$ & $0.063(1)$ \\
\hline$C(24)$ & $0.2847(2)$ & $0.2641(2)$ & $0.2320(2)$ & $0.073(1)$ \\
\hline$C(25)$ & $0.1548(2)$ & $0.2575(1)$ & $0.2557(2)$ & $0.060(0)$ \\
\hline$C(26)$ & $0.1673(3)$ & $0.1645(1)$ & $0.2994(2)$ & $0.078(1)$ \\
\hline$C(27)$ & $0.2943(2)$ & $0.1482(1)$ & $0.3555(2)$ & $0.076(1)$ \\
\hline$C(31)$ & $0.1943(1)$ & $0.4824(1)$ & $0.5007(1)$ & $0.044(0)$ \\
\hline$\alpha(32)$ & $0.1724(1)$ & $0.5796(1)$ & $0.4999(1)$ & $0.044(0)$ \\
\hline $\mathbf{N}$ & $0.1964(1)$ & $0.6181(1)$ & $0.6176(1)$ & $0.039(0)$ \\
\hline$C(33)$ & $0.1741(2)$ & $0.7122(1)$ & $0.6047(1)$ & $0.051(0)$ \\
\hline Q(34) & $0.1953(2)$ & $0.7556(1)$ & $0.7228(2)$ & $0.061(0)$ \\
\hline$C(35)$ & $0.3335(2)$ & $0.7376(1)$ & $0.8118(2)$ & $0.065(0)$ \\
\hline$Q(36)$ & $0.3587(2)$ & $0.6409(1)$ & $0.8221(2)$ & $0.061(0)$ \\
\hline$C(37)$ & $0.3325(1)$ & $0.6011(1)$ & $0.7008(1)$ & $0.050 \times 0)$ \\
\hline
\end{tabular}

äquivalenter isotroper Auslenkungsparameter, definiert als 1/3 der Spur des orthogonalisierten $U_{i}$ Tensors.
TABELLE 2. Ortskoordinaten und atomare Auslenkungsparameter $\left(\mathrm{pm}^{2}\right)$ der Nichtwasserstoff-Atome von endo-Sila-biperiden [rac(Si RS,C2SR)-endo-2]

\begin{tabular}{lrlll}
\hline Atom & \multicolumn{1}{l}{$\boldsymbol{x}$} & $y$ & $z$ & $U_{\text {eq }}{ }^{\mathrm{y}}$ \\
\hline $\mathrm{Si}$ & $0.2390(1)$ & $0.1298(1)$ & $0.7263(1)$ & $0.041(0)$ \\
$\mathrm{O}$ & $0.2755(2)$ & $0.0249(2)$ & $0.7680(2)$ & $0.060(1)$ \\
$\mathrm{C}(11)$ & $0.3257(2)$ & $0.1675(3)$ & $0.6662(2)$ & $0.043(1)$ \\
$\mathrm{C}(12)$ & $0.3871(2)$ & $0.0910(3)$ & $0.6518(2)$ & $0.059(1)$ \\
$\mathrm{C}(13)$ & $0.4528(3)$ & $0.1153(5)$ & $0.6071(2)$ & $0.075(1)$ \\
$\mathrm{C}(14)$ & $0.4577(3)$ & $0.2163(5)$ & $0.5760(2)$ & $0.074(1)$ \\
$\mathrm{C}(15)$ & $0.3969(3)$ & $0.2916(4)$ & $0.5885(2)$ & $0.076(1)$ \\
$\mathrm{C}(16)$ & $0.3324(3)$ & $0.2676(3)$ & $0.6336(2)$ & $0.061(1)$ \\
$\mathrm{C}(21)$ & $0.1456(2)$ & $0.0895(3)$ & $0.6746(2)$ & $0.049(1)$ \\
$\mathrm{C}(22)$ & $0.0693(2)$ & $0.0503(4)$ & $0.7170(2)$ & $0.067(1)$ \\
$\mathrm{C}(23)$ & $-0.0038(3)$ & $0.1147(5)$ & $0.6836(3)$ & $0.072(1)$ \\
$\mathrm{C}(24)$ & $0.0222(3)$ & $0.1144(4)$ & $0.6074(2)$ & $0.075(1)$ \\
$\mathrm{C}(25)$ & $0.1052(3)$ & $0.1734(4)$ & $0.6228(2)$ & $0.069(1)$ \\
$\mathrm{C}(26)$ & $0.0718(3)$ & $0.2661(4)$ & $0.6671(3)$ & $0.087(2)$ \\
$\mathrm{C}(27)$ & $0.0090(3)$ & $0.2291(5)$ & $0.7028(3)$ & $0.089(2)$ \\
$\mathrm{C}(31)$ & $0.2153(2)$ & $0.2462(3)$ & $0.7857(2)$ & $0.049(1)$ \\
$\mathrm{C}(32)$ & $0.2856(2)$ & $0.2794(3)$ & $0.8347(2)$ & $0.050(1)$ \\
$\mathrm{N}$ & $0.2716(2)$ & $0.3826(2)$ & $0.8715(1)$ & $0.041(1)$ \\
$\mathrm{C}(33)$ & $0.1986(2)$ & $0.3760(3)$ & $0.9172(2)$ & $0.051(1)$ \\
$\mathrm{C}(34)$ & $0.1839(2)$ & $0.4847(3)$ & $0.9537(2)$ & $0.059(1)$ \\
$\mathrm{C}(35)$ & $0.2599(3)$ & $0.5205(4)$ & $0.9955(2)$ & $0.070(1)$ \\
$\mathrm{C}(36)$ & $0.3360(3)$ & $0.5200(4)$ & $0.9487(2)$ & $0.062(1)$ \\
$\mathrm{C}(37)$ & $0.3455(2)$ & $0.4100(3)$ & $0.9125(2)$ & $0.056(1)$ \\
\hline
\end{tabular}

Äquivalenter isotroper Auslenkungsparameter, definiert als $1 / 3$ der Spur des orthogonalisierten $U_{i}$-Tensors.

den [Torsionswinkel Si-C(31)-C(32)-N: 171.5 bzw. 169.2 ${ }^{\circ}$.

Sila-biperiden und endo-Sila-biperiden bilden im Kristall intermolekulare $\mathrm{O}-\mathrm{H} \cdots \mathrm{N}$-WasserstoffBrückenbindungen aus (Sila-biperiden: $\mathrm{O} \cdots \mathrm{N} 281.0$,

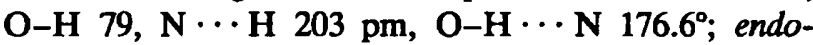
Sila-biperiden: $\mathrm{O} \cdots \mathrm{N} 274.5, \mathrm{O}-\mathrm{H} 96, \mathrm{~N} \cdots \mathrm{H} 182$ pm, $\left.\mathrm{O}-\mathrm{H} \cdots \mathrm{N} 162.0^{\circ}\right)$. Im Falle von Sila-biperiden (Abb. 3) führen diese zur Ausbildung von zentrosymmetrischen Dimeren, während im Kristall von endoSila-biperiden (Abb. 4) unendliche Ketten vorliegen. Diese unterschiedlichen Wasserstoff-Brückenbindungs-Muster implizieren auch unterschiedliche Orientierungen des freien Elektronenpaars am StickstoffAtom relativ zur OH-Gruppe des gleichen Moleküls (exo-Verbindung: $\mathrm{C}(31)-\mathrm{C}(32)-\mathrm{N}-\mathrm{C}(33)-62.5$, $\mathrm{C}(31)-\mathrm{C}(32)-\mathrm{N}-\mathrm{C}(37) \quad 175.7^{\circ}$; endo-Verbindung: $\mathrm{C}(31)-\mathrm{C}(32)-\mathrm{N}-\mathrm{C}(33) \quad 179.0, \quad \mathrm{C}(31)-\mathrm{C}(32)-\mathrm{N}-\mathrm{C}(37)$ $57.8^{\circ}$ ). Ein ähnliches Wasserstoff-BrückenbindungsSystem, wie es für Sila-biperiden beobachtet wurde, findet sich auch im Kristall des Racemats von Sila-procyclidin (10) [20].

\subsection{Pharmakologische Untersuchungen und Rezeptor- Bindungsstudien}

Alle im Rahmen der vorliegenden Arbeit untersuchten Verbindungen hemmten am Vas deferens des 
TABELLE 3. Bindungsabstände (pm) und -winkel $\left(^{\circ}\right)$ der Nichtwasserstoff-Atome von Sila-biperiden [ rac-(Si RS,C2SR)-exo-2]

\begin{tabular}{|c|c|c|c|}
\hline \multicolumn{4}{|l|}{ Abstände } \\
\hline $\mathbf{O}-\mathbf{S i}$ & $162.6(1)$ & $\mathrm{C}(11)-\mathrm{Si}$ & $187.2(2)$ \\
\hline$C(21)-S i$ & $187.9(2)$ & $C(31)-S i$ & $187.4(1)$ \\
\hline$C(12)-C(11)$ & $139.9(2)$ & $C(16)-C(11)$ & $139.6(2)$ \\
\hline$C(13)-C(12)$ & $138.3(3)$ & $C(14)-C(13)$ & $136.8(2)$ \\
\hline$C(15)-C(14)$ & $138.7(2)$ & $C(16)-C(15)$ & $138.5(3)$ \\
\hline$C(22)-C(21)$ & $155.7(2)$ & $C(25)-C(21)$ & $157.4(2)$ \\
\hline$C(23)-C(22)$ & $155.0(3)$ & $C(24)-C(23)$ & $151.5(3)$ \\
\hline$C(27)-C(23)$ & $150.0(3)$ & $C(25)-C(24)$ & $153.4(4)$ \\
\hline$C(26)-C(25)$ & $152.1(3)$ & $C(27)-C(26)$ & $131.0(3)$ \\
\hline$C(32)-C(31)$ & $152.1(2)$ & $\mathrm{N}-\mathrm{C}(32)$ & $147.4(2)$ \\
\hline$C(33)-N$ & $147.4(2)$ & $C(37)-N$ & $146.7(2)$ \\
\hline$C(34)-C(33)$ & $151.6(3)$ & $C(35)-C(34)$ & $150.9(2)$ \\
\hline$C(36)-C(35)$ & $151.7(3)$ & $C(37)-C(36)$ & $151.7(2)$ \\
\hline \multicolumn{4}{|l|}{ Winkel } \\
\hline$C(11)-S i-O$ & $105.9(1)$ & $\mathrm{C}(21)-\mathrm{Si}-\mathrm{O}$ & $111.6(1)$ \\
\hline$C(21)-S i-C(11)$ & $110.3(1)$ & $C(31)-S i-O$ & $111.4(1)$ \\
\hline$C(31)-S i-C(11)$ & $110.2(1)$ & $C(31)-S i-C(21)$ & $107.4(1)$ \\
\hline$C(12)-C(11)-S i$ & $122.4(1)$ & $C(16)-C(11)-S i$ & $120.4(1)$ \\
\hline$C(16)-C(11)-C(12)$ & $117.0(1)$ & $C(13)-C(12)-C(11)$ & $121.5(1)$ \\
\hline$C(14)-C(13)-C(12)$ & $120.2(1)$ & $C(15)-C(14)-C(13)$ & $119.9(2)$ \\
\hline$C(16)-C(15)-C(14)$ & $119.8(1)$ & $C(15)-C(16)-C(11)$ & $121.5(1)$ \\
\hline$C(22)-C(21)-S i$ & $115.7(1)$ & $C(25)-C(21)-S i$ & $116.7(1)$ \\
\hline$C(25)-C(21)-C(22)$ & $101.4(1)$ & $C(23)-C(22)-C(21)$ & $103.8(1)$ \\
\hline$C(24)-C(23)-C(22)$ & $99.5(2)$ & $C(27)-C(23)-C(22)$ & $107.5(2)$ \\
\hline$C(27)-C(23)-C(24)$ & $100.0(2)$ & $C(25)-C(24)-C(23)$ & $94.5(2)$ \\
\hline$C(24)-C(25)-C(21)$ & 101.1(1) & $C(26)-C(25)-C(21)$ & $104.9(2)$ \\
\hline$C(26)-C(25)-C(24)$ & $99.1(2)$ & $C(27)-C(26)-C(25)$ & $107.6(2)$ \\
\hline$C(26)-C(27)-C(23)$ & $108.2(2)$ & $C(32)-C(31)-S i$ & $114.8(1)$ \\
\hline$N-C(32)-C(31)$ & $114.9(1)$ & $C(33)-N-C(32)$ & $109.5(1)$ \\
\hline $\mathrm{C}(37)-\mathrm{N}-\mathrm{C}(32)$ & $111.4(1)$ & $C(37)-N-C(33)$ & 109.5(1) \\
\hline$C(34)-C(33)-N$ & 112.1(1) & $C(35)-C(34)-C(33)$ & $110.9(2)$ \\
\hline$C(36)-C(35)-C(34)$ & $109.9(1)$ & $C(37)-C(36)-C(35)$ & $111.0(1)$ \\
\hline$C(36)-C(37)-N$ & $111.8(1)$ & & \\
\hline
\end{tabular}

Kaninchens konzentrationsabhängig die durch den M1-selektiven Agonisten 4-Cl-McN-A-343 (- $\log$ EC $_{50}$ $=6.60 \pm 0.09 ; n=27$ ) induzierte Abnahme der neurogenen Kontraktionen. In gleicher Weise wurden die durch Arecaidinpropargylester ausgelösten negativ inotropen Effekte $\left(-\log \mathrm{EC}_{50}=8.07 \pm 0.08 ; n=47\right)$ am Meerschweinchen-Atrium sowie die Kontraktionen $\left(-\log \mathrm{EC}_{50}=7.59 \pm 0.09 ; n=51\right)$ des Meerschweinchen-Ileum antagonisiert. An allen drei Geweben verschoben Biperiden, Sila-biperiden und endo-Sila-biperiden die Agonisten-KonzentrationsWirkungs-Kurven parallel zu höheren Konzentrationen ohne nennenswerten Einfluß auf den Basaltonus der Organe und den Maximaleffekt der Agonisten. Die Arunlakshana-Schild-Darstellungen zeigten einen linearen Verlauf, und die Steigungen der Regressionsgeraden waren in keinem Fall von 1.00 verschieden $(P>$ 0.05). In allen Kompetitionsexperimenten konnte die Bindung der Antagonisten an die vier Muscarinrezeptor-Subtypen unter der Annahme einer einheitlichen Population von Bindungsstellen beschrieben werden.
So waren die Hill-Koeffizienten in keinem Fall signifikant von 1.00 verschieden $(P>0.05)$. Alle diese Befunde sprechen für einen rein kompetitiven Antagonismus an jeweils nur einer Bindungsstelle, so daß die erhaltenen $\mathrm{pA}_{2}$ - bzw. $\mathrm{pK}_{\mathrm{i}}$-Werte als echtes $\mathrm{MaB}$ für die Affinität der Antagonisten zu den Muscarin-Rezeptoren gelten können. Die Ergebnisse der pharmakologischen Experimente und Rezeptor-Bindungsstudien sind in den Tabellen 5 und 6 sowie in Abb. 5 zusammengefaßt. Aus Vergleichsgründen sind in beiden Tabellen sowie in Abb. 5 zusätzlich die der Literatur entnommenen $\mathbf{p A}_{2}-$ [13] bzw. $\mathrm{pK}_{\mathrm{i}}$-Werte [21] des M1-selektiven Antagonisten Pirenzepin aufgeführt.

Die in den funktionellen Experimenten bestimmten $\mathrm{pA}_{2}$-Werte (Tabelle 5) zeigen eine sehr gute Übereinstimmung mit den $\mathrm{pK}_{\mathrm{i}}$-Werten (Tabelle 6), die in den Radioligand-Kompetitionsexperimenten erhalten wurden (Abb. 5). Weiterhin bestätigen die im Rahmen dieser Arbeit erhaltenen Ergebnisse die von anderen

TABELLE 4. Bindungsabstände (pm) und -winkel $\left(^{\circ}\right)$ der Nichtwasserstoff-Atome von endo-Sila-biperiden [rac-(Si $R S, \mathrm{C} 2 S R$ )-ndo2]

\begin{tabular}{|c|c|c|c|}
\hline \multicolumn{4}{|l|}{ Abstände } \\
\hline $\mathbf{O}-\mathbf{S i}$ & $162.1(3)$ & $\mathbf{C}(11)-\mathrm{Si}$ & $186.7(4)$ \\
\hline $\mathrm{C}(21)-\mathrm{Si}$ & $186.8(4)$ & $\mathrm{C}(31)-\mathrm{Si}$ & $186.3(4)$ \\
\hline $\mathrm{C}(12)-\mathrm{C}(11)$ & $139.0(5)$ & $\alpha(16)-C(11)$ & $137.9(5)$ \\
\hline$C(13)-C(12)$ & $139.3(5)$ & $C(14)-C(13)$ & $137.5(7)$ \\
\hline$C(15)-C(14)$ & $136.6(6)$ & $C(16)-C(15)$ & $138.2(6)$ \\
\hline$C(22)-C(21)$ & $154.9(5)$ & $C(25)-C(21)$ & $156.8(5)$ \\
\hline$C(23)-C(22)$ & $155.4(6)$ & $C(24)-C(23)$ & $151.9(6)$ \\
\hline$C(27)-C(23)$ & $146.2(7)$ & $C(25)-C(24)$ & $154.8(6)$ \\
\hline$\alpha(26)-C(25)$ & $151.5(6)$ & $\alpha(27)-\alpha(26)$ & $130.3(7)$ \\
\hline$C(32)-C(31)$ & $152.5(5)$ & $\mathrm{N}-\mathrm{C}(32)$ & $146.4(4)$ \\
\hline $\mathbf{C ( 3 3 ) - N}$ & $146.9(4)$ & $\mathbf{C}(37)-\mathrm{N}$ & $146.5(4)$ \\
\hline$C(34)-C(33)$ & $152.1(5)$ & $C(35)-C(34)$ & $152.8(5)$ \\
\hline$C(36)-C(35)$ & $151.9(5)$ & $C(37)-C(36)$ & $152.1(5)$ \\
\hline \multicolumn{4}{|l|}{ Winkel } \\
\hline C(11)-Si-O & $103.2(2)$ & $\mathrm{C}(21)-\mathrm{Si}-\mathrm{O}$ & $110.1(2)$ \\
\hline $\mathrm{C}(21)-\mathrm{Si}-\mathrm{C}(11)$ & $109.9(2)$ & C(31)-Si-O & $112.3(2)$ \\
\hline$C(31)-S i-C(11)$ & $109.9(2)$ & $\mathrm{C}(31)-\mathrm{Si}-\mathrm{C}(21)$ & 111.1(2) \\
\hline$C(12)-C(11)-S i$ & $119.2(3)$ & $C(16)-C(11)-S i$ & $123.9(3)$ \\
\hline$\alpha(16)-\alpha(11)-\alpha(12)$ & $116.9(4)$ & $\alpha(13)-\alpha(12)-\alpha(11)$ & $121.3(4)$ \\
\hline$C(14)-C(13)-C(12)$ & $120.1(4)$ & $C(15)-\alpha(14)-\alpha(13)$ & $119.3(4)$ \\
\hline$C(16)-C(15)-C(14)$ & $120.4(4)$ & $C(15)-C(16)-C(11)$ & $122.0(4)$ \\
\hline$C(22)-C(21)-S i$ & $116.3(3)$ & $\mathrm{C}(25)-\mathrm{C}(21)-\mathrm{Si}$ & $119.7(3)$ \\
\hline$C(25)-C(21)-C(22)$ & $101.8(3)$ & $C(23)-C(22)-C(21)$ & $103.2(3)$ \\
\hline$C(24)-C(23)-C(22)$ & $100.7(3)$ & $C(27)-C(23)-C(22)$ & $106.0(4)$ \\
\hline$C(27)-C(23)-C(24)$ & $101.8(5)$ & $C(25)-C(24)-\alpha(23)$ & 93.1(3) \\
\hline$C(24)-C(25)-C(21)$ & $100.0(4)$ & $C(26)-C(25)-C(21)$ & $106.6(3)$ \\
\hline$C(26)-C(25)-C(24)$ & $98.6(4)$ & $C(27)-C(26)-C(25)$ & $108.0(4)$ \\
\hline$C(26)-C(27)-C(23)$ & $108.1(5)$ & $C(32)-C(31)-S i$ & $115.3(2)$ \\
\hline$N-C(32)-C(31)$ & $114.3(3)$ & $C(33)-N-C(32)$ & $111.3(3)$ \\
\hline $\mathrm{C}(37)-\mathrm{N}-\mathrm{C}(32)$ & $109.3(3)$ & $C(37)-N-C(33)$ & $110.1(3)$ \\
\hline$C(34)-C(33)-N$ & $110.6(3)$ & $C(35)-C(34)-C(33)$ & $111.5(3)$ \\
\hline$C(36)-C(35)-C(34)$ & $109.6(3)$ & $C(37)-C(36)-C(35)$ & $110.7(3)$ \\
\hline$C(36)-C(37)-N$ & $111.4(3)$ & & \\
\hline
\end{tabular}


TABELLE 5. Affinitäten ( $\mathrm{pA}_{2}$-Werte) und Steigungen in den Arunlakshana-Schild-Darstellungen (in Klammern) von Biperiden, Sila-biperiden und endo-Sila-biperiden an muscarinischen M1- (Vas deferens des Kaninchens), M2- (Meerschweinchen-Atrium) und M3-Rezeptoren (Meerschweinchen-lleum). Angegeben sind Mittelwerte \pm Standardfehler der Mittehwerte für 8-16 Einzelexperimente. Die Steigungen waren in keinem Falle signifikant von 1.00 verschieden $(P>0.05)$. Aus Vergleichsgründen sind zusätzlich die Ref. 13 entnommenen $\mathrm{pA}_{2}$-Werte des M1-selektiven Antagonisten Pirenzepin aufgefiuhrt.

\begin{tabular}{lccc}
\hline Antagonist & \multicolumn{3}{l}{$\mathrm{pA}_{2}$-Werte } \\
\cline { 2 - 4 } & $\mathrm{M} 1$ & $\mathrm{M} 2$ & $\mathrm{M} 3$ \\
\hline Biperiden & $8.80 \pm 0.03$ & $7.57 \pm 0.04$ & $8.14 \pm 0.03$ \\
& $(1.06 \pm 0.06)$ & $(1.06 \pm 0.07)$ & $(1.00 \pm 0.06)$ \\
Sila-biperiden & $8.79 \pm 0.02$ & $7.79 \pm 0.02$ & $8.21 \pm 0.02$ \\
& $(1.06 \pm 0.03)$ & $(1.01 \pm 0.04)$ & $(0.99 \pm 0.04)$ \\
endo-Sila-biperiden & $8.72 \pm 0.04$ & $7.73 \pm 0.06$ & $8.11 \pm 0.03$ \\
& $(1.00 \pm 0.07)$ & $(1.17 \pm 0.10)$ & $(0.98 \pm 0.05)$ \\
Pirenzepin & $8.24 \pm 0.06$ & $6.82 \pm 0.03$ & $6.88 \pm 0.04$ \\
\hline
\end{tabular}

Arbeitsgruppen bestimmten Affinitäten des Biperidens an nativen M1-, M2- und M3-Rezeptoren bzw. an klonierten humanen m1-, m2-, m3- und m4-Rezeptoren $[22,23]$.

Sowohl Biperiden als auch Sila-biperiden und endo-Sila-biperiden zeigten die höchste Affinität zu den M1- und die niedrigste Affinität (bis zu einem Faktor von 25: M1 > M2) zu den kardialen M2-Rezeptoren. Alle drei Verbindungen besitzen folgendes Affinitätsprofil: M1 > M4 > M3 > M2. Dieses Profil ist dem des Pirenzepins qualitativ sehr ähnlich (Tabellen 5 und 6; Abb. 5), jedoch sind die Affinitäten des Pirenzepins zu allen vier Muscarinrezeptor-Subtypen deutlich niedriger als die von Biperidin, Sila-biperiden und endo-Sila-biperiden.

Auffällig ist, daß die Sila-Substitution von Biperiden (C/Si-Austausch: $\rightarrow$ Sila-biperiden) keinen nennenswerten Einfluß auf die Affinität zu den einzelnen Muscarinrezeptor-Subtypen hat. Bemerkenswert ist

TABELLE 6. Affinitäten ( $\mathrm{pK}_{\mathrm{i}}$-Werte) von Biperiden, Sila-biperiden und endo-Sila-biperiden an muscarinischen M1- (NB-OK-1-Zellen), M2- (Rattenherz), M3-(Rattenpankreas) und M4-Rezeptoren (Rattenstriatum). Die $\mathrm{pK}_{\mathrm{i}}$-Werte wurden in Kompetitionsexperimenten unter Verwendung von $\left[{ }^{3} \mathrm{H}\right]-\mathrm{NMS}$ als Radioligand ermittelt. Angegeben sind die Mittelwerte aus mindestens drei voneinander unabhängigen Experimenten. Aus Vergleichsgründen sind zusätzlich die Ref. 21 entnommenen pK $_{\mathrm{i}}$-Werte des M1-selektiven Antagonisten Pirenzepin aufgeführt.

\begin{tabular}{lllll}
\hline Antagonist & \multicolumn{4}{l}{ pK $_{\text {-Werte }}$} \\
\cline { 2 - 5 } & $\mathbf{M} 1$ & M2 & M3 & M4 \\
\hline Biperiden & 9.1 & 7.7 & 8.1 & 8.4 \\
Sila-biperiden & 9.1 & 7.8 & 8.3 & 8.6 \\
endo-Sila-biperiden & 8.8 & 7.7 & 8.1 & 8.5 \\
Pirenzepin & 8.3 & 6.5 & 6.8 & 7.1 \\
\hline
\end{tabular}

$\mathrm{PA}_{2}-$ Worto: $\square M 1, \bullet M 2, \triangle M 3 / p K_{1}-$ Worto: $\square M 1, \circ M 2, \Delta M 3$

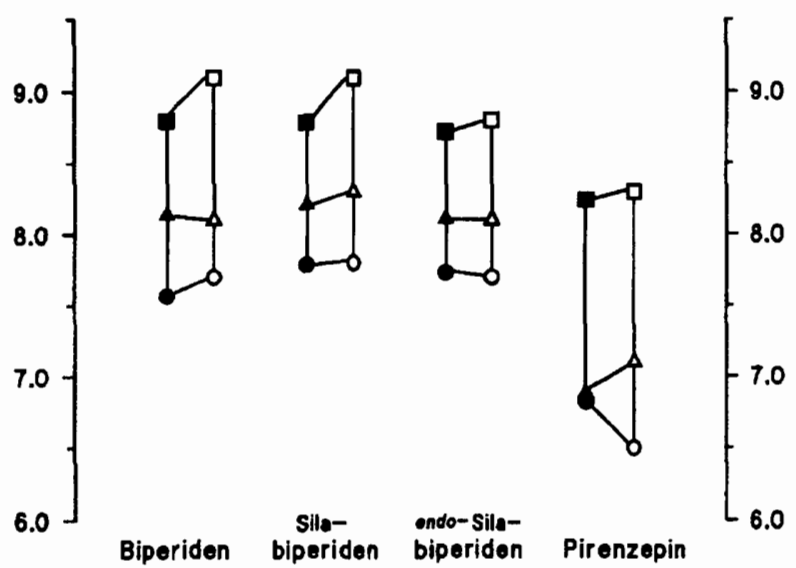

Abb. 5. Affinitätsprofile von Biperiden, Sila-biperiden, endo-Sila-biperiden und Pirenzepin an Muscarinrezeptor-Subtypen. Die $\mathrm{pA}_{2-}$ Werte (geschlossene Symbole; Tabelle 5) wurden in funktionellen Experimenten am Vas deferens des Kaninchens (M1-Rezeptoren) sowie am Atrium (M2-Rezeptoren) un Ileum (M3-Rezeptoren) des Meerschweinchens bestimmt. Die $\mathrm{pK}_{\mathrm{i}}$-Werte (offene Symbole; Tabelle 6) wurden in Bindungsstudien unter Verwendung von $\left[{ }^{3} \mathrm{H}\right]-$ NMS als Radioligand an M1- (NB-OK-1-Zellen), M2- (Rattenherz) und M3-Rezeptoren (Rattenpankreas) ermittelt.

auch, da $B$ sich die beiden diastereomeren SiliciumVerbindungen Sila-biperiden und endo-Sila-biperiden in ihren Affinitäten zu den Muscarin-Rezeptoren praktisch nicht unterscheiden. Es ist allerdings eine noch offene Frage, ob die beobachteten Struktur-WirkungsBeziehungen durch eine (zumindest partielle) Epimerisierung von Sila-biperiden und endo-Sila-biperiden zu rac-(Si $R S, C 2 R S)$-exo-2 bzw. rac-(Si $R S, C 2 R S)$-endo-2 beeinflußt werden (vgl. hierzu Abschnitt 2.1.).

\section{Experimenteller Teil}

\subsection{Synthesen}

Schmelzpunkte (nicht korrigiert): Kofler-Heiztischmikroskop der Fa. Reichert. ${ }^{1}$ H-NMR-Spektren [Lösungsmittel $\mathrm{CDCl}_{3}$, interner Standard $\mathrm{CHCl}_{3}(\delta=$ 7.25)]: AC-250- (250.1 MHz), AM-300- (300.1 MHz) und WM-400-Gerät $(400.1 \mathrm{MHz})$ der Fa. Bruker; zur Kennzeichnung der H-Atome siehe Abb. 6. ${ }^{13} \mathrm{C}$-NMRSpektren [Lösungsmittel und interner Standard $\mathrm{CDCl}_{3}$ $(\delta=77.05)]$ : AC-250- (62.9 MHz), AM-300- (75.5 MHz)

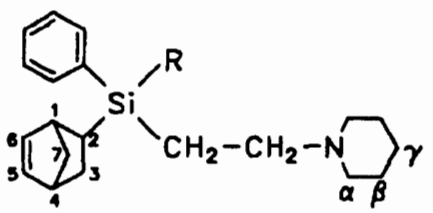

Abb. 6. Kennzeichnung der Atome für die Angabe der ${ }^{1} \mathrm{H}$ - und ${ }^{13} \mathrm{C}$-NMR-Daten $\left(\mathrm{R}=\mathrm{OCH}_{3}, \mathrm{OH}\right)$. 
und WM-400-Gerät (100.6 MHz) der Fa. Bruker; zur Kennzeichnung der C-Atome siehe Abb. 6. Die Signalzuordnungen wurden aus DEPT-Experimenten und im Falle der Endstufen aus ${ }^{1} \mathrm{H},{ }^{1} \mathrm{H}$ - und ${ }^{1} \mathrm{H},{ }^{13} \mathrm{C}$ COSY-Experimenten abgeleitet; die Ergebnisse dieser Experimente sind in den Zuordnungen enthalten. ${ }^{29} \mathrm{Si}$ NMR-Spektren [Lösungsmittel $\mathrm{CDCl}_{3}$, interner Standard TMS $(\delta=0)$ ]: AC-250-Gerät der Fa. Bruker (49.7 MHz). EI-MS-Spektren ( $70 \mathrm{eV}$ ): Gerät Finnigan-MAT711. Die angegebenen $m / z$-Werte für die Molekülionen und intensitätsstärksten Fragmentionen basieren auf den Massenzahlen der jeweiligen Isotope mit der größten natürlichen relativen Häufigkeit $\left({ }^{1} \mathrm{H},{ }^{12} \mathrm{C},{ }^{14} \mathrm{~N}\right.$, $\left.{ }^{16} \mathrm{O},{ }^{28} \mathrm{Si}\right)$. Alle Synthesen wurden unter Feuchtigkeitsausschluß in getrockneten Lösungsmitteln (Siedebereich des verwendeten Petrolethers $40-65^{\circ} \mathrm{C}$ ) unter Stickstoff durchgeführt.

3.1.1. rac-(SiRS,C2RS)-(endo-Bicyclo[2.2.1]hept-5en-2-yl)phenyl(2-piperidinoethyl)silanol / rac-(SiRS, C2SR)-(endo-Bicyclo[2.2.1]hept-5-en-2-yl)phenyl(2-piperidinoethyl)silanol / rac-(SiRS,C2RS)-(exo-Bicyclo[2.2.1]hept-5-en-2-yl)phenyl(2-piperidinoethyl)silanol / rac-(SiRS,C2SR)-(exo-Bicyclo[2.2.1]hept-5-en-2-yl)phenyl(2-piperidinoethyl)silanol Irac-(SiRS,C2RS)-endo2 / rac-(SiRS,C2SR)-endo-2 / rac-(SiRS,C2RS)-exo-2 / rac-(SiRS,C2SR)-exo-2]

$\mathrm{Zu}$ einer Lösung von $20.9 \mathrm{~g}$ (61.2 mmol) rac-(Si $R S$, C2RS)endo-9/rac-(Si $R S, C 2 S R$ )endo-9/rac-(Si $R S$, C2RS)-exo-9/rac-(Si $R S, C 2 S R$ )exo-9 $(7: 7: 3: 3)$ in 400 ml 2-Propanol wurden bei Raumtemperatur unter Rühren $100 \mathrm{ml}$ einer $0.1 \mathrm{M}$ wässerigen $\mathrm{KOH}$-Lösung gegeben. Man rührte $3 \mathrm{~d}$ bei Raumtemperatur, befreite unter vermindertem Druck bei $40^{\circ} \mathrm{C}$ von den leichtflüchtigen Anteilen, nahm den Rückstand in 300 $\mathrm{ml}$ Diethylether auf, wusch fünfmal mit jeweils $30 \mathrm{ml}$ Wasser, trocknete die etherische Lösung über Magnesiumsulfat und entfernte im Vakuum das Lösungsmittel. Ausb. $18.6 \mathrm{~g}$ (93\%) eines Feststoffes, der sich aus einem Gemisch aus rac-( $\mathrm{Si} R S, \mathrm{C} 2 R S)$ endo-2/rac(Si $R S, C 2 S R$ )endo-2/rac-(Si $R S, C 2 R S$ ) exo-2 und rac(Si RS,C2SR)exo-2 (Mol-Verhältnis $7: 7: 3: 3,{ }^{13} \mathrm{C}$ NMR) zusammensetzte. Durch fraktionierende Kristallisation dieses Gemisches $\left({ }^{13} \mathrm{C}-\mathrm{NMR}-\mathrm{Kontrolle}\right)$ wurden rac-(Si $R S, \mathrm{C} 2 S R)$-endo-2 und rac-(Si $R S, \mathrm{C} 2 S R)$ exo-2 rein isoliert (siehe nachfolgende Angaben).

3.1.1.1. Gewinnung von endo-Sila-biperiden /rac(SiRS, C2SR)-endo-2] $63.2 \mathrm{~g}$ eines Gemisches aus rac(Si RS, C2RS)-endo-2/rac-(Si RS,C2SR)-endo-2/rac(Si $R S, C 2 R S$ )-exo-2 und rac-(Si $R S, C 2 S R$ )-exo-2 $(7: 7: 3: 3)$ wurden in $120 \mathrm{ml}$ Diethylether $/ n$-Hexan $(1 / 1, v / v)$ gelöst. Man ließ diese Lösung $14 \mathrm{~d}$ bei $-10^{\circ} \mathrm{C}$ ungestört stehen, isolierte die ausgefallenen Kristalle $(19.7 \mathrm{~g})$ durch Filtration und kristallisierte sechsmal aus Diethylether um (Kristallisation durch langsames Verdunsten des Lösungsmittels bei Raumtemperatur). Ausb. $5.2 \mathrm{~g}$ weiße, nadelförmige Kristalle; Schmp. $95-98^{\circ} \mathrm{C} .{ }^{1} \mathrm{H}-\mathrm{NMR}(400 \mathrm{MHz}): \delta 0.78$ (ddd, $\left.1 \mathrm{H},{ }^{2} J=15.5 \mathrm{~Hz},{ }^{3} \mathrm{~J}=5.6 \mathrm{~Hz},{ }^{3} \mathrm{~J}=5.6 \mathrm{~Hz}, \mathrm{SiCH}_{2}\right) ; 1.07$ (ddd, $1 \mathrm{H},{ }^{2} J=15.5 \mathrm{~Hz},{ }^{3} J=6.3 \mathrm{~Hz},{ }^{3} J=9.5 \mathrm{~Hz}$, $\mathrm{SiCH}_{2}$ ); 1.13 (ddd, $1 \mathrm{H},{ }^{2} J_{7,7^{\prime}}=7.8 \mathrm{~Hz},{ }^{3} J_{1,7}=1.6 \mathrm{~Hz}$, $\left.{ }^{3} J_{4,7}=1.6 \mathrm{~Hz}, \mathrm{H} 7\right) ; 1.16\left(\mathrm{ddd}, 1 \mathrm{H},{ }^{2} J_{3 \text { endo,3exo }}=11.3\right.$ $\mathrm{Hz},{ }^{3} J_{2, \text { Bendo }}=5.6 \mathrm{~Hz},{ }^{4} J_{\text {3endo. }} 7^{\prime}=1.9 \mathrm{~Hz}$, endo $-\mathrm{H} 3$ ); 1.37 (ddd, $1 \mathrm{H},{ }^{3} J_{1,2}=2.9 \mathrm{~Hz},{ }^{3} J_{2,3 e n d o}=5.6 \mathrm{~Hz},{ }^{3} J_{2,3 e \times o}$ $=9.6 \mathrm{~Hz}, \mathrm{H} 2) ; 1.36-1.40\left(\mathrm{~m}, 1 \mathrm{H}, \mathrm{H}^{\prime}\right) ; 1.39-1.46(\mathrm{~m}$, $2 \mathrm{H}, \gamma-\mathrm{H}) ; 1.51-1.62(\mathrm{~m}, 4 \mathrm{H}, \beta-\mathrm{H}) ; 1.91$ (ddd, $1 \mathrm{H}$, ${ }^{2} J_{3 \text { endo }, 3 \text { exo }}=11.3 \mathrm{~Hz},{ }^{3} J_{2,3 \text { exo }}=9.6 \mathrm{~Hz},{ }^{3} J_{3 \text { exo. } 4}=3.8$ $\mathrm{Hz}$, exo-H3); 2.1-2.6 (m, 6H, $\left.\mathrm{NCH}_{2}, \alpha-\mathrm{H}\right) ; 2.88$ (breites "s", 1H, H4); 2.98 (breites "s", 1H, H1); 5.95 (dd, 1H, $\left.{ }^{3} J_{1,6}=2.8 \mathrm{~Hz},{ }^{3} J_{5,6}=5.6 \mathrm{~Hz}, \mathrm{H6}\right) ; 5.99\left(\mathrm{dd}, 1 \mathrm{H},{ }^{3} J_{4,5}=\right.$ $\left.3.0 \mathrm{~Hz},{ }^{3} J_{5,6}=5.6 \mathrm{~Hz}, \mathrm{H} 5\right) ; 7.27-7.36$ und $7.56-7.62(\mathrm{~m}$, $3 \mathrm{H}$ bzw. $\left.2 \mathrm{H}, \mathrm{SiC}_{6} \mathrm{H}_{5}\right) .{ }^{13} \mathrm{C}-\mathrm{NMR}: \delta 11.2\left(\mathrm{SiCH}_{2}\right) ; 24.2$ $(\gamma-\mathrm{C}) ; 25.78$ (C2); 25.83 ( $\beta$-C); 26.8 (C3); 42.2 (C4); 44.2 (C1); $51.2(\mathrm{C} 7) ; 54.1(\alpha-\mathrm{C}) ; 54.6\left(\mathrm{NCH}_{2}\right) ; 127.5$ (m-C); 128.8 (p-C); $133.4(o-\mathrm{C}) ; 134.0(\mathrm{CH}) ; 135.5$ (CH); 138.9 (i-C). ${ }^{29}$ Si-NMR: $\delta$ 5.4. EI-MS: $m / z 327$ $\left(10 \%, \mathrm{M}^{+}\right)$; $98\left(100 \%, \mathrm{CH}_{2}=\mathrm{NC}_{5} \mathrm{H}_{10}^{+}\right)$. Anal. Gef.: C, 73.9; $\mathrm{H}, 9.0 ; \mathrm{Si}, 8.8 . \mathrm{C}_{20} \mathrm{H}_{29} \mathrm{NOSi}(327.5)$ ber.: C, 73.34; $\mathrm{H}, 8.92 ; \mathrm{Si}, 8.57 \%$.

3.1.1.2. Gewinnung von Sila-biperiden Irac-(SiRS, C2SR)-exo-2] Ein Teil der bei der oben beschriebenen Gewinnung von rac-( $\mathrm{Si} R S, \mathrm{C} 2 S R$ )-endo-2 durch Umkristallisieren aus Diethylether anfallenden Mutterlaugen wurde vom Lösungsmittel befreit, und man erhielt $2.72 \mathrm{~g}$ eines Feststoffes, der sich aus $20 \%$ rac(SiRS,C2RS)-endo-2, 25\% rac-(Si $R S, C 2 S R$ )endo-2, $20 \%$ rac-(Si $R S, C 2 R S)$-exo-2 und $35 \%$ rac(Si $R S, C 2 S R)$-exo-2 $\left({ }^{13} \mathrm{C}-\mathrm{NMR}\right)$ zusammensetzte. Man löste diesen Feststoff in $8 \mathrm{ml}$ Diethylether, ließ die resultierende Lösung $3 \mathrm{~d}$ bei Raumtemperatur ungestört stehen und isolierte die ausgefallenen Kristalle $(2.24 \mathrm{~g})$ durch Filtration. Die größeren, blättchenförmigen Kristalle $(1.62 \mathrm{~g})$ wurden durch mechanische Auslese von den kleineren, nadelförmigen Kristallen $(0.62 \mathrm{~g})$ abgetrennt und vierzehnmal aus Diethylether umkristallisiert (Kristallisation durch langsames Abkühlen einer siedenden Lösung auf Raumtemperatur). Ausb. $40 \mathrm{mg}$ farblose Kristalle,

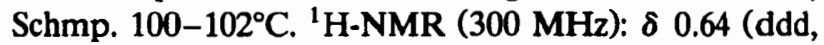
$1 \mathrm{H},{ }^{3} J_{1,2}=1.8 \mathrm{~Hz},{ }^{3} J_{2,3 \text { endo }}=9.7 \mathrm{~Hz},{ }^{3} J_{2,3 e x o}=5.8 \mathrm{~Hz}$, $\mathrm{H} 2) ; 0.90-0.99\left(\mathrm{~m}, 1 \mathrm{H}, \mathrm{SiCH}_{2}\right) ; 1.03-1.27(\mathrm{~m}, 4 \mathrm{H}$, endo- $\mathrm{H} 3, \mathrm{H} 7, \mathrm{H}^{\prime}$ und $1 \mathrm{H}$ von $\left.\mathrm{SiCH}_{2}\right) ; 1.44-1.70(\mathrm{~m}$, $6 \mathrm{H}, \beta-\mathrm{H}, \gamma-\mathrm{H}) ; 1.72\left(\mathrm{ddd}, 1 \mathrm{H},{ }^{2} J_{3 \text { endo,3exo }}=11.3 \mathrm{~Hz}\right.$, ${ }^{3} J_{2,3 \text { exo }}=5.8 \mathrm{~Hz},{ }^{3} J_{3 \text { exo, } 4}=3.5 \mathrm{~Hz}$, exo-H3); $2.19-2.68$ (m, 6H, $\left.\mathrm{NCH}_{2}, \alpha-\mathrm{H}\right) ; 2.88$ (breites "s", $\left.1 \mathrm{H}, \mathrm{H} 4\right) ; 2.95-$ 
$3.00(\mathrm{~m}, 1 \mathrm{H}, \mathrm{H} 1) ; 5.94\left(\mathrm{dd}, 1 \mathrm{H},{ }^{3} J_{4,5}=3.0 \mathrm{~Hz},{ }^{3} J_{5,6}=5.6\right.$ $\mathrm{Hz}, \mathrm{H} 5) ; 6.16\left(\mathrm{dd}, 1 \mathrm{H},{ }^{3} J_{1,6}=3.0 \mathrm{~Hz},{ }^{3} J_{5,6}=5.6 \mathrm{~Hz}\right.$, H6); 7.26-7.38 und 7.54-7.66 (m, $3 \mathrm{H}$ bzw. $2 \mathrm{H}, \mathrm{SiC}_{6} \mathrm{H}_{5}$ ). ${ }^{13}$ C-NMR: $\delta 10.9\left(\mathrm{SiCH}_{2}\right) ; 24.3(\gamma-\mathrm{C}) ; 25.0(\mathrm{C} 2) ; 26.1$ $(\beta-\mathrm{C}) ; 26.5$ (C3); 42.5 (CH); $42.9(\mathrm{CH}) ; 46.9(\mathrm{C7}) ; 54.3$ $(\alpha-\mathrm{C}) ; 54.9\left(\mathrm{NCH}_{2}\right) ; 127.7(m-\mathrm{C}) ; 129.1(p-\mathrm{C}) ; 133.6$ (o-C); $134.1(\mathrm{CH}) ; 137.9(\mathrm{CH}) ; 138.7(i-\mathrm{C}) .{ }^{29} \mathrm{Si}-\mathrm{NMR}$ : $\delta$ 5.1. EI-MS: $m / z \quad 327\left(10 \%, M^{+}\right) ; 98(100 \%$, $\mathrm{CH}_{2}=\mathrm{NC}_{5} \mathrm{H}_{10}^{+}$). Anal. Gef.: $\mathrm{C}, 73.7 ; \mathrm{H}, 9.0 ; \mathrm{Si}, 8.6$. $\mathrm{C}_{20} \mathrm{H}_{29} \mathrm{NOSi}$ (327.5) ber.: C, 73.34; $\mathrm{H}, 8.92 ; \mathrm{Si}, 8.57 \%$.

\subsubsection{Trichlor(vinyl)silan (3)}

Trichlor(vinyl)silan (3) stand als Handelsprodukt (Merck) zur Verfügung.

3.1.3. rac-(C2RS)-(endo-Bicyclo[2.2.1] hept-5-en-2yl) trichlorsilan / rac-(C2RS)-(exo-Bicyclo[ 2.2.1] hept-5en-2-yl) trichlorsilan (rac-(C2RS)-endo-4 / rac-(C2RS)exo-4]

Ein Gemisch aus rac-(C2RS)-endo-4 und rac(C2RS) exo-4 (Mol-Verhältnis 7:3, ${ }^{1} \mathrm{H}-\mathrm{NMR}$ ) wurde gemäß Ref. 19 hergestellt; Ausb. 89\%.

3.1.4. rac-(C2RS)-(endo-Bicyclo[2.2.1]hept-5-en-2yl)trimethoxysilan / rac-(C2RS)-(exo-Bicyclo[2.2.1]hept5-en-2-yl)trimethoxysilan [rac-(C2RS)-endo-5 / rac(C2RS)-exo-5]

$\mathrm{Zu}$ einer Lösung von $352 \mathrm{~g}(1.55 \mathrm{~mol}) \mathrm{rac}-(\mathrm{C} 2 R S)$ endo-4/rac-(C2RS)-exo-4 $(7: 3)$ und $475 \mathrm{~g}(4.69 \mathrm{~mol})$ Triethylamin in $6 \mathrm{~L}$ Petrolether wurden bei Raumtemperatur innerhalb von $\mathbf{4 5}$ min unter kräftigem Rühren $155 \mathrm{~g}$ (4.84 mol) Methanol getropft. Man rührte $20 \mathrm{~min}$ bei Raumtemperatur, filtrierte den Niederschlag ab, wusch diesen mit Petrolether, vereinigte Filtrat und Waschlösung, befreite unter vermindertem Druck vom Lösungsmittel und destillierte den Rückstand im Vakuum fraktionierend über eine Vigreux-Kolonne. Ausb. 277 g (84\%) einer farblosen Flüssigkeit; Sdp. $50-53^{\circ} \mathrm{C} / 0.1$ Torr; endo / exo-Verhältnis $7: 3\left({ }^{1} \mathrm{H}\right.$ NMR). ${ }^{1} \mathrm{H}-\mathrm{NMR}$ (endo / exo, $300 \mathrm{MHz}$ ): $\delta$ 0.4-0.5, 1.0-1.5 und 1.7-2.1 (m, 5H, H2, H3, H7); 2.9-3.0 (m, $2 \mathrm{H}, \mathrm{H1}, \mathrm{H} 4)$; 3.53 (endo) und 3.60 (exo) (s, $9 \mathrm{H}, \mathrm{OCH}_{3}$ ); 6.0-6.2 (m, 2H, H5, H6). ${ }^{13} \mathrm{C}-\mathrm{NMR}$ (endo): $\delta 20.1$ (C2); 26.7 (C3); 42.0 (CH); $44.0(\mathrm{CH}) ; 50.3\left(\mathrm{OCH}_{3}\right)$; 50.7 (C7); $134.6(\mathrm{CH}) ; 135.3(\mathrm{CH}) .{ }^{13} \mathrm{C}-\mathrm{NMR}$ (exo): $\delta$ 19.5 (C2); 26.0 (C3); 42.4 (CH); 42.6 (CH); 47.0 (C7); $50.5\left(\mathrm{OCH}_{3}\right) ; 133.6(\mathrm{CH}) ; 137.4(\mathrm{CH}) .{ }^{29} \mathrm{Si}-\mathrm{NMR}$ (endo): $\delta-17.8 .{ }^{29} \mathrm{Si}-\mathrm{NMR}($ exo): $\delta-17.2$. EI-MS: $\mathrm{m} / z 214\left(45 \%, \mathrm{M}^{+}\right) ; 121\left(100 \%, \mathrm{M}^{+}-\mathrm{C}_{7} \mathrm{H}_{9}\right)$. Anal. Gef.: C, 56.1; $\mathrm{H}, 8.5 ; \mathrm{Si}$, 13.0. $\mathrm{C}_{10} \mathrm{H}_{18} \mathrm{O}_{3} \mathrm{Si}(214.3)$ ber.: $\mathrm{C}, 56.04 ; \mathrm{H}, 8.46 ; \mathrm{Si}, 13.10 \%$.
3.1.5. $\mathrm{rac}-(\mathrm{C} 2 \mathrm{RS})$-(endo-Bicyclo[2.2.1]hept-5-en-2yl)dimethoxy (phenyl)silan / rac-(C2RS)-(exo-Bicyclo[2.2.1]hept-5-en-2-yl)dimethoxy(phenyl)silan [rac(C2RS)-endo-6 / rac-(C2RS)-exo-6]

Ein aus $16.5 \mathrm{~g}(0.68 \mathrm{~mol})$ Magnesium und $107 \mathrm{~g}$ $(0.68 \mathrm{~mol})$ Brombenzol in $300 \mathrm{ml}$ Diethylether hergestelltes Grignard-Reagenz wurde bei $0^{\circ} \mathrm{C}$ innerhalb von $1.5 \mathrm{~h}$ unter kräftigem Rühren zu einer Lösung von $122 \mathrm{~g}(0.57 \mathrm{~mol}) \mathrm{rac}-(\mathrm{C} 2 \mathrm{RS})$-endo-5/rac-(C2RS)exo-5 (7:3) in $300 \mathrm{ml}$ Diethylether getropft. Man rührte $2 \mathrm{~h}$ bei Raumtemperatur, filtrierte den Niederschlag $a b$, wusch diesen mit Petrolether, vereinigte Filtrat und Waschlösung, entfernte das Lösungsmittel unter vermindertem Druck und destillierte den Rückstand im Vakuum fraktionierend über eine Vigreux-Kolonne. Ausb. 137 g (92\%) einer farblosen Flüssigkeit; Sdp. $113-116^{\circ} \mathrm{C} / 0.1$ Torr; endo / exo-Verhältnis 7:3 $\left({ }^{1} \mathrm{H}\right.$ NMR). ${ }^{1} \mathrm{H}-\mathrm{NMR}$ (endo / exo, $250 \mathrm{MHz}$ ): $\delta$ 0.7-0.8, 1.0-1.5 und 1.8-2.0 (m, 5H, H2, H3, H7); 2.8-3.0 (m, $2 \mathrm{H}, \mathrm{H1}, \mathrm{H} 4) ; 3.58$ und 3.59 (endo) sowie 3.646 und 3.648 (exo) (s, 6H, $\mathrm{OCH}_{3}$ ); 5.9-6.1 (m, 2H, H5, H6); 7.3-7.7 (m, 5H, $\mathrm{SiC}_{6} \mathrm{H}_{5}$ ). ${ }^{13} \mathrm{C}-\mathrm{NMR}$ (endo): $\delta 23.2$ (C2); 26.9 (C3); $42.2(\mathrm{CH}) ; 44.2(\mathrm{CH}) ; 50.65$ und 50.69 $\left(\mathrm{OCH}_{3}\right) ; 50.9$ (C7); 127.7 (m-C); 129.7 (p-C); 133.5 (i-C); 134.5 (o-C); $134.6(\mathrm{CH}) ; 135.2(\mathrm{CH}) .{ }^{13} \mathrm{C}-\mathrm{NMR}$ (exo): $\delta 22.4(\mathrm{C} 2) ; 26.2$ (C3); $42.5(\mathrm{CH}) ; 42.7(\mathrm{CH}) ; 46.9$ (C7); $50.9\left(\mathrm{OCH}_{3}\right) ; 127.9(m-\mathrm{C}) ; 130.0(p-\mathrm{C}) ; 132.9$ (i-C); $133.8(\mathrm{CH}) ; 134.6$ (o-C); $137.8(\mathrm{CH}) .{ }^{29} \mathrm{Si}-\mathrm{NMR}$ (endo): $\delta$ - 17.8. ${ }^{29} \mathrm{Si}-\mathrm{NMR}$ (exo): $\delta$ - 17.3. EI-MS: $m / z 260\left(23 \%, M^{+}\right) ; 167\left(100 \%, M^{+}-\mathrm{C}_{7} \mathrm{H}_{9}\right)$. Anal. Gef.: C, 68.8; $\mathrm{H}, 7.7 ; \mathrm{Si}, 10.8 . \mathrm{C}_{15} \mathrm{H}_{20} \mathrm{O}_{2} \mathrm{Si}(260.4)$ ber.: C, 69.19; H, 7.74; Si, $10.79 \%$.

3.1.0. rac-(SiRS,C2RS)-(endo-Bicyclo[2.2.1]hept-5en-2-yl) phenyl(vinyl)silan / rac-(SiRS,C2SR)-(endo-Bicyclo[2.2.1] hept-5-en-2-yl)phenyl(vinyl)silan / rac-(SiRS, C2RS)-(exo-Bicyclo[2.2.1Jhept-5-en-2-yl)phenyl(vinyl)silan / rac-(SiRS,C2SR)-(exo-Bicyclo[ 2.2.1]hept-5-en-2yl)phenyl(vinyl)silan [rac-(SiRS, C2RS)-endo-7 / rac(SiRS,C2SR)-endo-7 / rac-(SiRS,C2RS)-exo-7 / rac(Si-RS,C2SR)-exo-7]

$\mathrm{Zu}$ einer Lösung von $50.1 \mathrm{~g}(0.19 \mathrm{~mol}) \mathrm{rac}-(\mathrm{C} 2 R S)$ endo-6/rac-(C2RS)-exo-6 (7:3) in $200 \mathrm{ml}$ Diethylether wurden unter Rühren bei $0^{\circ} \mathrm{C}$ innerhalb von $2 \mathrm{~h} 115 \mathrm{ml}$ einer $1.75 \mathrm{M}$ Lösung von Vinylmagnesiumchlorid in Tetrahydrofuran $\left(0.2 \mathrm{~mol} \mathrm{CH}_{2}=\mathrm{CHMgCl}\right)$ getropft. Man rührte $2 \mathrm{~h}$ bei Raumtemperatur, filtrierte den Niederschlag $a b$, befreite das Filtrat unter vermindertem Druck vom Lösungsmittel, versetzte das Filtrat mit $n$-Hexan, filtrierte erneut, befreite das Filtrat unter vermindertem Druck vom Lösungsmittel und destillierte den Rückstand im Vakuum fraktionierend über eine Vigreux-Kolonne. Es wurden $42.6 \mathrm{~g}$ einer farb- 
losen Flüssigkeit (Sdp. 94-96 ${ }^{\circ} \mathrm{C} / 0.05$ Torr) erhalten. $40.2 \mathrm{~g}$ dieser Flüssigkeit wurden in $300 \mathrm{ml}$ Diethylether gelöst und bei Raumtemperatur unter Rühren mit 6.10 g (0.16 mol) Lithiumaluminiumhydrid versetzt. Man rührte $18 \mathrm{~h}$ unter Rückfluß, filtrierte den Niederschlag ab, wusch diesen zweimal mit jeweils $50 \mathrm{ml}$ Diethylether, vereinigte Filtrat und Waschlösungen und tropfte zu dieser Lösung bei Raumtemperatur unter kräftigem Rühren $100 \mathrm{ml}$ einer $10 \%$ igen wässerigen Ammoniumhydrochlorid-Lösung. Anschließend trennte man die etherische Phase ab, trocknete diese über Magnesiumsulfat, entfernte unter vermindertem Druck das Lösungsmittel und destillierte den Rückstand im Vakuum fraktionierend über eine Vigreux-Kolonne. Ausb. $21.5 \mathrm{~g}(52 \%)$ einer farblosen Flüssigkeit; Sdp. $88-91^{\circ} \mathrm{C} / 0.1$ Torr; Mol-Verhältnis rac-(Si RS,C2RS)endo-7/rac-(Si RS,C2SR)-endo-7/rac-(Si RS,C2RS)exo-7/rac-(SiRS,C2SR)-exo-7 7:7:3:3 ( $\left.{ }^{1} \mathrm{H}-\mathrm{NMR}\right)$. ${ }^{1} \mathrm{H}-\mathrm{NMR}$ (endo / exo, $300 \mathrm{MHz}$ ): $\delta$ 0.7-0.8 und 0.9-2.1 (m, 5H, H2, H3, H7); 2.9-3.2 (m, 2H, H1, H4); 4.3-4.4 (endo) und 4.6-4.7 (exo) $(\mathrm{m}, 1 \mathrm{H}, \mathrm{SiH}) ; 5.9-6.5(\mathrm{~m}, 5 \mathrm{H}$, $\left.\mathrm{SiCH}=\mathrm{CH}_{2}, \mathrm{H} 5, \mathrm{H} 6\right) ; 7.4-7.7\left(\mathrm{~m}, 5 \mathrm{H}, \mathrm{SiC}_{6} \mathrm{H}_{5}\right) .{ }^{13} \mathrm{C}-$ NMR (endo): $\delta$ 22.15/22.19 (C2); 28.0/28.1 (C3); 42.46/42.51 (CH); 44.83/44.87 (CH); 51.0 (C7); 127.8/127.9 (m-C); 129.27/129.30 ( $p-\mathrm{C}) ; 133.95$ (CH); $134.1 / 134.2$ (CH); 134.8/135.0 (i-C); 135.0/135.1 (oC); $135.0 / 135.2\left(\mathrm{SiCH}=\mathrm{CH}_{2}\right) ; 135.8 / 135.9(\mathrm{CH}) .{ }^{13} \mathrm{C}-$ NMR (exo): $\delta$ 21.4 (C2); 28.1/28.3 (C3); 42.96/43.00 (CH); 43.55/43.61 (CH); 46.7 (C7); 127.9/128.0 (m-C); $129.4 / 129.5$ ( $p-\mathrm{C}) ; 133.5 / 133.7(\mathrm{CH}) ; 133.95 / 134.04$ (CH); $134.45 / 134.65$ (i-C); $135.11 / 135.14$ (o-C); $135.6 / 135.7\left(\mathrm{SiCH}=\mathrm{CH}_{2}\right) ; 137.68 / 137.72(\mathrm{CH}){ }^{29} \mathrm{Si}-$ NMR (endo): $\delta-12.35 /-13.01 .{ }^{29} \mathrm{Si}-\mathrm{NMR}$ (exo): $\delta$ $-10.98 /-11.14$. EI-MS: $m / z 226\left(12 \%, M^{+}\right) ; 159$ (100\%, $\mathrm{C}_{10} \mathrm{H}_{11} \mathrm{Si}^{+}$). Anal. Gef.: C, 79.7; H, 8.0; Si, 12.5. $\mathrm{C}_{15} \mathrm{H}_{18} \mathrm{Si}$ (226.4) ber.: C, 79.58; H, 8.01; Si, $12.41 \%$.

3.1.7. rac-(SiRS, C2RS)-(endo-Bicyclo[2.2.1]hept-5en-2-yl))phenyl(piperidino)(2-piperidinoethyl)silan / rac(SiRS,C2SR)-(endo-Bicyclo[2.2.1]hept-5-en-2-yl)phenyl(piperidino) (2-piperidinoethyl)silan / rac-(SiRS,C2RS)(exo-Bicyclo[2.2.1]hept-5-en-2-yl)phenyl(piperidino)(2piperidinoethyl)silan / rac-(SiRS,C2SR)-(exo-Bicyclo[2.2.1]hept-5-en-2-yl)phenyl (piperidino) (2-piperidinoethyl)silan / rac-(SiRS, C2RS)-endo-8/rac-(SiRS, C2SR)-endo-8 / rac-(SiRS, C2RS)-exo-8 / rac-(SiRS, C2SR)-exo-8]

$\mathrm{Zu}$ einer Lösung von $103 \mathrm{~g}(1.21 \mathrm{~mol})$ Piperidin in $280 \mathrm{ml}$ Tetrahydrofuran wurden unter Rühren bei $40^{\circ} \mathrm{C}$ innerhalb von $40 \mathrm{~min} 125 \mathrm{ml}$ einer $1.6 \mathrm{M}$ n-Butyllithium-Lösung in Hexan (0.20 mol n-BuLi) getropft. Dann rührte man $30 \mathrm{~min}$ bei $40^{\circ} \mathrm{C}$, versetzte innerhalb von $1 \mathrm{~h}$ tropfenweise mit einer Lösung von $77.5 \mathrm{~g}(0.34$ mol) rac-(Si $R S, \mathrm{C} 2 R S)$-endo-7/rac-(Si $R S, \mathrm{C} 2 S R$ )endo-7/rac-(Si $R S, C 2 R S$ )-exo-7/rac-(Si $R S, C 2 S R$ )-exo-7 $(7: 7: 3: 3)$ in $200 \mathrm{ml}$ Tetrahydrofuran und rührte weitere $3 \mathrm{~h}$ bei $40^{\circ} \mathrm{C}$ und $12 \mathrm{~h}$ bei Raumtemperatur. $\mathrm{Zu}$ diesem Reaktionsgemisch tropfte man eine Lösung von $135 \mathrm{~g}$ (1.33 mol) Triethylamin und $56.1 \mathrm{~g}(0.52 \mathrm{~mol})$ Chlortrimethylsilan in $130 \mathrm{ml}$ Diethylether, rührte $4 \mathrm{~h}$ bei Raumtemperatur, filtrierte den Niederschlag ab, wusch diesen mit $150 \mathrm{ml}$ Diethylether, vereinigte Filtrat und Waschlösung und befreite unter vermindertem Druck vom Lösungsmittel. Der Rückstand wurde mit $300 \mathrm{ml} \mathrm{n}$-Hexan versetzt, und man filtrierte erneut. Das Filtrat wurde unter vermindertem Druck vom Lösungsmittel befreit und der Rückstand in 10-gPortionen im Kugelrohrverdampfer $\left(200^{\circ} \mathrm{C} / 0.05\right.$ Torr $)$ destilliert. Ausb. $111.1 \mathrm{~g}$ (82\%) einer farblosen Flüssigkeit; Mol-Verhältnis rac-(Si $R S, \mathrm{C} 2 R S)$-endo-8/ rac-(Si $R S, C 2 S R)$-endo-8/rac-(Si $R S, C 2 R S$ )-exo-8/rac(Si RS,C2SR)-exo-8 7:7:3:3 ( $\left.{ }^{13} \mathrm{C}-\mathrm{NMR}\right) .{ }^{1} \mathrm{H}-\mathrm{NMR}$ (endo / exo, $250 \mathrm{MHz}$ ): $\delta$ 0.6-3.1 (m, 31H, aliphat. $\mathrm{H}$ ); 5.8-6.3 (m, 2H, olefin. H); 6.2-7.7 (m, 5H, $\left.\mathrm{SiC}_{6} \mathrm{H}_{5}\right)$. ${ }^{29} \mathrm{Si}-\mathrm{NMR}$ (endo): $\delta-1.84 /-1.22 .{ }^{29} \mathrm{Si}-\mathrm{NMR}$ (exo): $\delta$ $-1.03 /-1.25$. EI-MS: $m / z 394\left(<1 \%, \mathrm{M}^{+}\right) ; 309$ (100\%). Anal. Gef.: C, 75.9; H, 10.1; N, 6.9; Si, 7.2. $\mathrm{C}_{25} \mathrm{H}_{38} \mathrm{~N}_{2} \mathrm{Si}(394.7)$ ber.: $\mathrm{C}, 76.08 ; \mathrm{H}, 9.70 ; \mathrm{N}, 71.0 ; \mathrm{Si}$, $7.12 \%$

3.1.8. rac-(SiRS, C2RS)-(endo-Bicyclo[2.2.1Jhept-5en-2-yl)methoxy(phenyl)(2-piperidinoethyl)silan / rac-(SiRS,C2SR)-(endo-Bicyclo[2.2.1]hept-5-en-2-yl)methoxy(phenyl)(2-piperidinoethyl)silan / rac-(SiRS,C2RS)(exo-Bicyclo[2.2.1]hept-5-en-2-yl)methoxy (phenyl)(2-piperidinoethyl)silan / rac-(SiRS,C2SR)-(exo-Bicyclo/2.2. 1]hept-5-en-2-yl)methoxy (phenyl)(2-piperidinoethyl)silan [rac-(SiRS,C2RS)-endo-9 / rac-(SiRS,C2SR)-endo9/rac-(SiRS, C2RS)-exo-9 / rac-(SiRS, C2SR)-exo-9]

Eine Mischung aus $111 \mathrm{~g}(0.28 \mathrm{~mol}) \mathrm{rac}-(\mathrm{Si} R S$, $\mathrm{C} 2 \mathrm{RS}$ )-endo-8/rac-(Si $R S, \mathrm{C} 2 \mathrm{SR}$ )-endo-8/rac-(Si $R S$, C2RS)-exo-8/rac-(Si RS,C2SR)-exo-8 (7:7:3:3) und $30 \mathrm{ml}(0.74 \mathrm{~mol})$ Methanol wurde $20 \mathrm{~h}$ bei $60^{\circ} \mathrm{C}$ gerührt. Anschließend entfernte man unter vermindertem Druck die leichtflüchtigen Bestandteile und destillierte den Rückstand zunächst in 10-g-Portionen im Kugelrohrverdampfer und sodann über eine Vigreux-Kolonne. Ausb. $90.6 \mathrm{~g}(94 \%)$ einer farblosen Flüssigkeit; Sdp. $157-160^{\circ} \mathrm{C} / 0.01$ Torr; Mol-Verhältnis rac-(Si $R S$, $\mathrm{C} 2 R S$ )-endo-9/rac-(Si $R S, C 2 S R$ )-endo-9/rac-(SiRS, C2RS)-exo-9/rac-(Si $R S, C 2 S R)$ exo-9 $7: 7: 3: 3$ ( ${ }^{1} \mathrm{H}$ NMR). ${ }^{1} \mathrm{H}-\mathrm{NMR}$ (endo / exo, $\left.300 \mathrm{MHz}\right): \delta$ 0.6-3.0 (m, $21 \mathrm{H}$, aliphat. $\mathrm{H}$ ); $3.46 / 3.50$ (endo) und $3.538 / 3.544$ (exo) $\left(\mathrm{s}, 3 \mathrm{H}, \mathrm{OCH}_{3}\right) ; 5.9-6.1(\mathrm{~m}, 2 \mathrm{H}$, olefin. $\mathrm{H}) ; 7.3-7.6$ (m, 5H, $\mathrm{SiC}_{6} \mathrm{H}_{5}$ ). ${ }^{13} \mathrm{C}-\mathrm{NMR}$ (endo): $\delta 11.4 / 11.7$ $\left(\mathrm{SiCH}_{2}\right) ; 24.5(\gamma-\mathrm{C}) ; 24.60 / 24.64(\mathrm{C} 2) ; 26.0$ ( $\left.\beta-\mathrm{C}\right)$; 27.2/27.3 (C3); 42.27/42.29 (C4); 44.4/44.5 (C1); 51.2 
(C7); 51.27/51.31 $\left(\mathrm{OCH}_{3}\right) ; 53.8\left(\mathrm{NCH}_{2}\right) ; 54.0(\alpha-\mathrm{C})$; $127.59 / 127.62(m-C) ; 129.20 / 129.24(p-C) ; 134.0$ (oC); $134.3 / 134.4(\mathrm{CH}) ; 135.4 / 135.5(\mathrm{CH}) ; 136.0 / 136.1$ (i-C). ${ }^{13} \mathrm{C}$-NMR (exo): $\delta$ 11.0/11.2 $\left(\mathrm{SiCH}_{2}\right) ; 23.6 / 23.7$ (C2); 24.5 ( $\gamma$-C); 26.0 ( $\beta$-C); 26.4/26.5 (C3); 42.4/42.5 (CH); 42.7/42.8 (CH); 46.8/46.9 (C7); $51.4\left(\mathrm{OCH}_{3}\right)$; $53.7\left(\mathrm{NCH}_{2}\right) ; 54.0(\alpha-\mathrm{C}) ; 127.7(m-\mathrm{C}) ; 129.4(p-\mathrm{C})$; 133.6/133.7 (CH); 134.1 (o-C); $135.3 / 135.5$ (i-C); 138.0 (CH). ${ }^{29} \mathrm{Si}-\mathrm{NMR}$ (endo): $\delta 6.3 / 6.5 .{ }^{29} \mathrm{Si}-\mathrm{NMR}$ (exo): $\delta$ 6.63/6.64. EI-MS: $m / z 341\left(84 \%, \mathrm{M}^{+}\right)$; $98(100 \%$, $\mathrm{CH}_{2}=\mathrm{NC}_{5} \mathrm{H}_{10}^{+}$). Anal. Gef.: C, 73.8; $\mathrm{H}, 9.2 ; \mathrm{N}, 4.3 ; \mathrm{Si}$, 8.3. $\mathrm{C}_{21} \mathrm{H}_{31} \mathrm{NOSi}(341.6)$ ber.: $\mathrm{C}, 73.85 ; \mathrm{H}, 9.15 ; \mathrm{N}$, $4.10 ; \mathrm{Si}, 8.22 \%$.

\subsection{Kristallstrukturanalysen}

\subsubsection{Sila-biperiden [rac-(SiRS,C2SR)-exo-2]}

Sila-biperiden kristallisiert aus Diethylether in der monoklinen Raumgruppe $P 2_{1} / c ; a=1078.98(10), b=$ 1546.67(13), $c=1207.79(9) \mathrm{pm}, \beta=111.579(8)^{\circ}, Z=4$, $d_{\text {ber }}=1.208 \mathrm{~g} \mathrm{~cm}^{-3}$. Die Beugungsintensitäten wurden bei $20^{\circ} \mathrm{C}$ auf einem Syntex-Vierkreisdiffraktometer (Typ $P 2_{1}$ ) unter Verwendung von Graphit-monochromatisierter $\mathrm{Cu}-\mathrm{K} \alpha$-Strahlung $(\lambda=154.178 \mathrm{pm})$ im $\theta$ $2 \theta$-Betrieb $\left(3^{\circ} \leq 2 \theta \leq 135^{\circ}\right)$ gemessen. Die Meßgeschwindigkeit variierte in Abhängigkeit von der Reflexintensität zwischen $2.93^{\circ} \mathrm{min}^{-1}$ und $29.30^{\circ} \mathrm{min}^{-1}$. Die Daten wurden bezüglich Lorentz- und Polarisationseffekten korrigiert, eine Absorptionskorrektur wurde jedoch nicht durchgeführt $\left(\mu=1.037 \mathrm{~mm}^{-1}\right)$. Die Struktur wurde mit Direkten Methoden und Differenz-Fourier-Synthesen gelöst. Für die Verfeinerung wurden 3116 von 3360 gemessenen unabhängigen Reflexen herangezogen $(I \geq 2.5 \sigma(I))$. Die Verfeinerung konvergierte bei $R=0.040\left(R_{\mathrm{w}}=0.046\right)$. Eine abschließende Differenz-Fourier-Synthese ergab für die größten Elektronendichte-Maxima Werte von $0.26 \times 10^{-6} \mathrm{e}$ $\mathrm{pm}^{-3}$. Neben eigenen Programmen wurde das Programm SHELX-76 [24] verwendet. Es wurden komplexe Atomformfaktoren [25] verwendet.

\subsection{2. endo-Sila-biperiden ( $r a c-(S i R S, C 2 S R)$-endo-2]}

endo-Sila-biperiden kristallisiert aus Diethylether in der orthorhombischen Raumgruppe Pbca; $a=$ 1610.69(7), $b=1223.73(4), c=1914.63(9) \mathrm{pm}, Z=8$, $d_{\text {ber }}=1.153 \mathrm{~g} \mathrm{~cm}^{-3}$. Die Beugungsintensitäten wurden bei $20^{\circ} \mathrm{C}$ auf einem Syntex-Vierkreisdiffraktometer (Typ $P 2_{1}$ ) unter Verwendung von Graphit-monochromatisierter Mo-K $\alpha$-Strahlung $(\lambda=71.069 \mathrm{pm})$ im $\theta$ $2 \theta$-Betrieb $\left(3^{\circ} \leq 2 \theta \leq 50^{\circ}\right)$ gemessen. Die Meßgeschwindigkeit variierte in Abhängigkeit von der Reflexintensität zwischen $2.93^{\circ} \mathrm{min}^{-1}$ und $29.30^{\circ} \mathrm{min}^{-1}$. Die Daten wurden bezüglich Lorentz- und Polarisationseffekten korrigiert, eine Absorptionskorrektur wurde jedoch nicht durchgeführt $\left(\mu=0.094 \mathrm{~mm}^{-1}\right)$. Die Struktur wurde mit Direkten Methoden und Differenz-Fourier-Synthesen gelöst. Für die Verfeinerung wurden 2337 von 3337 gemessenen unabhängigen Reflexen herangezogen $(I \geq 2.5 \sigma(I))$. Die Verfeinerung konvergierte bei $R=0.074\left(R_{\mathrm{w}}=0.068\right)$. Eine abschließende Differenz-Fourier-Synthese ergab für die größten Elektronendichte-Maxima Werte von $0.32 \times$ $10^{-6} \mathrm{e} \mathrm{pm}^{-3}$. Neben eigenen Programmen wurde das Programm SHELX-76 [24] verwendet. Es wurden komplexe Atomformfaktoren [25] benutzt.

Weitere Einzelheiten zu den Kristallstrukturanalysen können beim Fachinformationszentrum Karlsruhe, Gesellschaft für wissenschaftlich-technische Information mbH, D-76344 Eggenstein-Leopoldshafen, unter Angabe der Hinterlegungsnummer CSD-57432, der Autorennamen und des Zeitschriftenzitats angefordert werden.

\subsection{Pharmakologische Untersuchungen}

\subsubsection{Vas deferens des Kaninchens}

Die Untersuchungen am Vas deferens des Kaninchens erfolgten weitgehend nach der in Ref. 26 beschriebenen Methodik. Männliche weiße New-Zealand-Kaninchen wurden durch i.v.-Injektion von Pentobarbital-Natrium $\left(120 \mathrm{mg} \mathrm{Kg}^{-1}\right)$ getötet. Die Vasa deferentia wurden entnommen und Segmente von 1.5 cm Länge in einem 6-ml-Organbad an einem isometrischen DMS-Aufnehmer fixiert. Als Badflüssigkeit [pH 7.4; Begasung mit $\left.\mathrm{O}_{2} / \mathrm{CO}_{2}(95 / 5, \mathrm{v} / \mathrm{v})\right]$ diente ein modifizierter Krebs-Puffer $\left(\mathrm{Ca}^{2+}\right.$-Konzentration $1.0 \mathrm{mM}$ ), der zur Blockade präsynaptischer $\alpha_{2}$-Rezeptoren Yohimbin $(1.0 \mu \mathrm{M})$ enthielt. Nach einer Äquilibrierungszeit von $30 \mathrm{~min}$ (Vorspannung $750 \mathrm{mg}$ ) wurden durch kontinuierliche Feldstimulation $0.5 \mathrm{~ms}$, $40 \mathrm{~V}, 0.05 \mathrm{~Hz}$ ) neurogene Einzelkontraktionen ausgelöst und auf einem Mehrkanalschreiber registriert. Diese Twitchkontraktionen wurden durch den M1-selektiven Agonisten [4-(4-Chlorphenylcarbamoyloxy)-2-butinyl]trimethylammoniumiodid (4-ClMcN-A343) Konzentrations-abhängig gehemmt.

\subsubsection{Vorhof und Ileum des Meerschweinchens}

Meerschweinchen beiderlei Geschlechts wurden durch Genickschlag getötet und aus den Carotiden entblutet. Der linke Vorhof und Streifen der Longitudinalmuskulatur des Ileum wurden in 6-ml-Organbäder überführt und unter einer Vorspannung von $500 \mathrm{mg}$ an DMS-Aufnehmern befestigt. Als Badflüssigkeit diente eine mit $\mathrm{O}_{2} / \mathrm{CO}_{2}(95 / 5, \mathrm{v} / \mathrm{v})$ durchströmte TyrodeLösung (pH 7.4, $32^{\circ} \mathrm{C}$ ). Die Vorhöfe wurden elektrisch stimuliert ( $3 \mathrm{~ms}, 5 \mathrm{~V}, 2 \mathrm{~Hz}$ ). Als Agonist wurde Are- 
caidinpropargylester verwendet. Die durch den Agonisten am Vorhof ausgelösten negativ inotropen Effekte wurden unter isometrischen und die Kontraktionen der Ileum-Muskulatur unter isotonischen Bedingungen auf einem Mehrkanalschreiber registriert. Für weitere experimentelle Details siehe Ref. 13.

\subsubsection{Affinität der Antagonisten}

Mit den oben genannten Agonisten wurden an den drei Geweben kumulative Konzentrations-WirkungsKurven in Ab- und Anwesenheit der Antagonisten erstellt und die $\mathrm{EC}_{50}$-Werte der Agonisten graphisch ermittelt (halblogarithmische Darstellung). Es wurden jeweils 3-5 Antagonisten-Konzentrationen (log Konzentrationsdifferenz $=0.5 ; n=4-6$ ) getestet (Inkubationszeit 30-60 min). Die Bestimmung der antimuscarinischen Potenz ( $\mathrm{pA}_{2}$-Werte) erfolgte gemäß Ref. 27. Da die Steigungen der Regressionsgeraden in den Arunlakshana-Schild-Darstellungen in keinem Fall von dem für einen kompetitiven Antagonismus theoretisch zu fordernden Wert von 1.00 signifikant abwichen, wurden die $\mathrm{pA}_{2}$-Werte der Antagonisten als Abszissenabschnitt der Regressionsgerade mit festgelegter Steigung von 1.00 ("constrained plot") berechnet [28].

Die pharmakologischen Kenngrößen sind in Tabelle 5 als Mittelwerte \pm Standardfehler der Mittelwerte angegeben. Die linearen Regressionsanalysen wurden nach der Methode der kleinsten Abweichungsquadrate durchgefuihrt. Die Prüfung auf Signifikanz des Unterschiedes zweier Mittelwerte erfolgte mit dem t-Test nach Student [28] auf einem Signifikanzniveau von $P<0.05$.

\subsubsection{Pharmaka}

Yohimbinhydrochlorid wurde von der Firma Sigma (Deisenhofen, Deutschland) bezogen. Arecaidinpropargylester [29] und 4-Cl-McN-A-343 [30] wurden nach Literaturvorschriften synthetisiert.

\subsection{Rezeptor-Bindungsstudien}

Die Radioligand-Kompetitionsexperimente wurden mit Homogenaten von NB-OK-1-Zellen (M1-Rezeptoren) sowie mit Homogenaten des Rattenherzens (M2-Rezeptoren), Rattenpankreas (M3-Rezeptoren) und Rattenstriatum (M4-Rezeptoren) in einem Natriumphosphat-Puffer $\left(50 \mathrm{mM}, 25^{\circ} \mathrm{C}\right)$ durchgeführt, der zusätzlich $\left[{ }^{3} \mathrm{H}\right]-\mathrm{N}-$ Methylscopolamin $\left(\left[{ }^{3} \mathrm{H}\right]-\mathrm{NMS}\right)$, steigende Konzentrationen der zu testenden Antagonisten sowie $2 \mathrm{mM} \mathrm{MgCl}_{2}$ enthielt (Gesamtvolumen $1.2 \mathrm{ml}$ ). Bei den Experimenten mit Homogenaten des Rattenpankreas enthielt der Inkubationspuffer außerdem 1\% Rinderserum-Albumin. Im Folgenden werden die experimentellen Bedingungen und die Auswertung kurz beschrieben. Für weitere experimentelle Details siehe Ref. 31 und Ref. 32.

\subsubsection{NB-OK-1-Zellen}

$80 \mu \mathrm{l}$ des Zellhomogenates wurden $2 \mathrm{~h}$ mit $0.25 \mathrm{nM}$ [ ${ }^{3} \mathrm{H}$ ]-NMS (entsprechend $2 \times \mathrm{K}_{\mathrm{D}}$ des Radioliganden) und steigenden Antagonisten-Konzentrationen inkubiert. Der Proteingehalt von $80 \mu 1$ Homogenat betrug etwa $200 \mu \mathrm{g}$.

\subsubsection{Rattenherz}

$80 \mu \mathrm{l}$ des Herzhomogenates (entsprechend 400-500 $\mu \mathrm{g}$ Protein) wurden $2 \mathrm{~h}$ mit $1.0 \mathrm{nM}\left[{ }^{3} \mathrm{H}\right.$-NMS (entsprechend $2 \times K_{D}$ des Radioliganden) und steigenden Antagonisten-Konzentrationen inkubiert.

\subsubsection{Rattenpankreas}

$1.0 \mathrm{ml}$ (entsprechend 800-1000 $\mu \mathrm{g}$ Protein) Pankreashomogenat wurde $4 \mathrm{~h}$ mit $0.24 \mathrm{nM}\left[{ }^{3} \mathrm{H}\right]-\mathrm{NMS}$ (entsprechend $2 \times \mathrm{K}_{\mathrm{D}}$ des Radioliganden an den M3Rezeptoren des Pankreas) und steigenden Antagonisten-Konzentrationen inkubiert.

\subsubsection{Rattenstriatum}

Im Rattenstriatum bindet $\left[{ }^{3} \mathrm{H}\right]-\mathrm{NMS}$ an vier Muscarinrezeptor-Subtypen (M1-M4). Um die ausschließ liche Bindung an die M4-Rezeptoren zu bestimmen, wurde die Tatsache ausgenutzt, daB $\left[{ }^{3} \mathrm{H}\right]-\mathrm{NMS}$ wesentlich schneller von den M1- und M2-Rezeptoren dissoziiert als von den M4- und M3-Rezeptoren [31,32]. Es wurde wie folgt vorgegangen.

$80 \mu \mathrm{l}$ des Striatumhomogenates (entsprechend 30 $\mu g$ Protein) wurden 2 h mit $0.24 \mathrm{nM}\left[{ }^{3} \mathrm{H}\right]-\mathrm{NMS}$ und steigenden Konzentrationen der zu testenden Antagonisten inkubiert. Dann wurde Atropin $(1 \mu \mathrm{M}$; dies entspricht etwa $1000 \times K_{D}$ von Atropin) hinzugegeben, 35 min gewartet und sodann filtriert. In früheren Kontrolluntersuchungen konnte gezeigt werden, daB nach der Dissoziationsperiode von $35 \mathrm{~min}$ der Radioligand 85\% M4- und 15\% M3-Rezeptoren besetzt [31,32]. Bei den im Rahmen dieser Arbeit durchgeführten Kompetitionsexperimenten störte die geringe $\left[{ }^{3} \mathrm{H}\right]-\mathrm{NMS}-\mathrm{Be}$ setzung der M3-Rezeptoren von $15 \%$ die exakte Ermittlung der M4-Affinitäten nicht. Die eingesetzte $\left[{ }^{3} \mathrm{H}\right]-N M S-K o n z e n t r a t i o n(0.24 \mathrm{nM})$ entspricht etwa 5 $\times K_{D}$ von $\left[{ }^{3} \mathrm{H}\right]-\mathrm{NMS}$ an den M4-Rezeptoren.

Am Ende der Inkubationszeit wurde mit $2 \mathrm{ml}$ eiskaltem Natriumphosphat-Puffer $(50 \mathrm{mM}, \mathrm{pH} 7.4)$ versetzt und das gebundene vom freien $\left[{ }^{3} \mathrm{H}\right]-\mathrm{NMS}$ durch schnelle Filtration über Whatman-GF/C-Glasmikrofaser-Filter getrennt. Alle Filter wurden vor ihrem Einsatz über Nacht mit $0.05 \%$ iger wässeriger Polyethylenimin-Lösung behandelt. Nach der Filtration wurden die Filter zum Auswaschen des ungebundenen 
$\left[{ }^{3} \mathrm{H}\right]-\mathrm{NMS}$ dreimal mit eiskalter Puffer-Lösung (siehe oben) gewaschen und dann getrocknet. Die Bestimmung der gebundenen Radioaktivität erfolgte in einem Szintillationszähler.

Die unspezifische $\left[{ }^{3} \mathrm{H}\right]-\mathrm{NMS}$-Bindung wurde in Kontrollexperimenten bei Anwesenheit von Atropin (1 $\mu \mathrm{M})$ ermittelt. Die Bestimmung der Proteinkonzentration in den Homogenaten erfolgte gemäß Ref. 33 unter Verwendung von Rinderserum-Albumin als Referenzsubstanz. Alle Kompetitionsexperimente wurden mindestens dreimal als Doppelbestimmungen durchgeführt.

\subsubsection{Auswertung}

Die Auswertung aller Kompetitionsexperimente erfolgte durch nichtlineare iterative Kurvenanpassung mittels eines in Ref. 34 beschriebenen Computerprogramms. Aus den so erhaltenen $\mathrm{IC}_{50}$-Werten wurden gemäß Ref. 35 die entsprechenden GleichgewichtsDissoziationskonstanten $\left(\mathbf{K}_{\mathrm{i}}\right.$-Werte) der Antagonisten berechnet. Die in Tabelle 6 zusammengestellten $\mathrm{pK}_{\mathrm{i}}$ Werte entsprechen $-\log K_{i}$. Die Standardabweichungen aller $\mathrm{pK}_{\mathrm{i}}$-Werte betrugen $\leq 0.15$ log-Einheiten ( $\leq 40 \%$ bei den $\mathrm{K}_{\mathrm{i}}$-Werten).

\subsubsection{Pharmaka}

[N-Methyl- ${ }^{3} \mathrm{H}$ ]scopolamin-chlorid $\left(\left[{ }^{3} \mathrm{H}\right.\right.$-NMS; etwa $80 \mathrm{Ci} / \mathrm{mmol}$ ) wurde von Amersham International (Bucks, England) und Atropinsulfat von Sigma Chemical Co. (St. Louis, MS, USA) bezogen.

\section{Dank}

Diese Arbeit wurde von der Deutschen Forschungsgemeinschaft und vom Fonds der Chemischen Industrie gefördert sowie durch Chemikalienspenden der Bayer AG (Leverkusen und Wuppertal-Elberfeld) unterstützt. J.P. dankt der Alexander von Humboldt-Stiftung und J.G. der Firma Boehringer Ingelheim für ein Forschungsstipendium.

\section{Literatur}

1 R. Tacke, M. Strecker, W.S. Sheldrick, E. Heeg, B. Berndt und K.M. Knapstein, Z. Naturforsch, 34b (1979) 1279.

2 L. Steiling, R. Tacke und U. Wannagat, Liebigs Ann. Chem., (1979) 1554.

3 R. Tacke, M. Strecker, W.S. Sheldrick, L. Ernst, E. Heeg, B. Berndt, C.-M. Knapstein und R. Niedner, Chem. Ber., 113 (1980) 1962.

4 R. Tacke, M. Strecker, G. Lambrecht, U. Moser und E. Mutschler, Liebigs Ann. Chem., (1983) 922.

5 R. Tacke, M. Strecker, G. Lambrecht, U. Moser und E. Mutschler, Arch. Pharm. (Weinheim), 317 (1984) 207.

6 R. Tacke, H. Linoh, H. Zilch, J. Wess, U. Moser, E. Mutschler und G. Lambrecht, Liebigs Ann. Chem., (1985) 2223.
7 R. Tacke und H. Zilch, Endeavour, New Series, 10 (1986) 191.

8 R. Tacke, J. Pikies, H. Linoh, R. Rohr-Aehle und S. Gönne, Liebigs Ann. Chem., (1987) 51.

9 R. Tacke, H. Linoh, L. Ernst, U. Moser, E. Mutschler, S. Sarge, H.K. Cammenga und G. Lambrecht, Chem. Ber., 120 (1987) 1229.

10 G. Lambrecht, R. Feifel, B. Forth, C. Strohmann, R. Tacke und E. Mutschler, Eur. J. Pharmacol, 152 (1988) 193.

11 M. Eltze, G. Gmelin, J. Wess, C. Strohmann, R. Tacke, E. Mutschler und G. Lambrecht, Eur. J. Pharmacol, 158 (1988) 233.

12 M. Waelbroeck, M. Tastenoy, J. Camus, J. Christophe, C. Strohmann, H. Linoh, H. Zilch, R. Tacke, E. Mutschler und G. Lambrecht, Br. J. Pharmacol., 98 (1989) 197.

13 G. Lambrecht, R. Feifel, M. Wagner-Röder, C. Strohmann, H. Zilch, R. Tacke, M. Waelbroeck, J. Christophe, H. Boddeke und E. Mutschler, Eur. J. Pharmacol, 168 (1989) 71.

14 G. Lambrecht, J. Wess, R. Tacke und E. Mutschler, in H. van der Goot, G. Domany, L. Pallos und H. Timmerman (Hrsg.), Trends in Medicinal Chemistry '88, Elsevier Science Publishers, Amsterdam, 1989, S. 265-282.

15 G. Lambrecht, R. Feifel, U. Moser, M. Wagner-Röder, L.K. Choo, J. Camus, M. Tastenoy, M. Waelbroeck, C. Strohmann, R. Tacke, J.F. Rodrigues de Miranda, J. Christophe und E. Mutschler, Trends Pharmacol. Sci. (Suppl.), 10 (1989) 65.

16 R. Tacke, K. Mahner, C. Strohmann, B. Forth, E. Mutschler, T. Friebe und G. Lambrecht, J. Organomet. Chem., 417 (1991) 339.

17 H.-P. Bächtold, A. Fürst, T. Struller und H. Kreiskott, in E. Bartholomé, E. Biekert, H. Hellman, H. Ley, W.M. Weigert und E. Weise (Hrsg.), Ullmanns Enzyklopädie der Technischen Chemie, 4. Aufl., Bd. 21, Verlag Chemie, Weinheim, 1982, S. 619-631.

18 W. Birkmayer und P. Riederer, Die Parkinson-Krankheit: Biochemie, Klinik, Therapie, 2. Aufl., Springer-Verlag, Wien, 1985.

19 R.F. Cunico, J. Org. Chem., 36 (1971) 929.

20 W.S. Sheldrick, H. Linoh, R. Tacke, G. Lambrecht, U. Moser und E. Mutschler, J. Chem. Soc., Dalton Trans., (1985) 1743.

21 M. Waelbroeck, M. Tastenoy, J. Camus und J. Christophe, Mol. Pharmacol., 38 (1990) 267.

22 M. Eltze und V. Figala, Eur. J. Pharmacol, 158 (1988) 11.

23 C. Bolden, B. Cusack und E. Richelson, J. Pharmacol. Exp. Ther., 260 (1992) 576.

24 G.M. Sheldrick, unveröffentlichte Ergebnisse.

25 D.T. Cromer und J.T. Waber, in J.A. Ibers und W.C. Hamilton (Hrsg.), International Tables for Crystallography, Bd. IV, Kynoch Press, Birmingham, 1974, S. 99-102 und S. 149.

26 M. Eltze, Eur. J. Pharmacol., 151 (1988) 205.

27 O. Arunlakshana und H.O. Schild, Br. J. Pharmacol, 14 (1959) 48.

28 R.J. Tallarida und R.B. Murray, Manual of pharmacologic calculations with computer programs, Springer-Verlag, Berlin, 1986.

29 U. Moser, G. Lambrecht, M. Wagner, J. Wess und E. Mutschler, Br. J. Pharmacol., 96 (1989) 319.

30 W.L. Nelson, D.S. Freeman und F.F. Vincenzi, J. Med. Chem., 19 (1976) 153.

31 M. Waelbroeck, J. Camus, M. Tastenoy, E. Mutschler, C. Strohmann, R. Tacke, L. Schjelderup, A. Aasen, G. Lambrecht und J. Christophe, Eur. J. Pharmacol. - MoL Pharmacol. Sect., 227 (1992) 33.

32 M. Waelbroeck, M. Tastenoy, J. Camus und J. Christophe, Mol Pharmacol., 38 (1990) 267.

33 O.H. Lowry, N.J. Rosebrough, A.L. Farr und R.J. Randall, J. Biol. Chem., 193 (1951) 265.

34 A. Richardson und A. Humrich, Trends Pharmacol. Sci, 5 (1984) 47.

35 Y. Cheng und W.H. Prusoff, Biochem. Pharmacol, 22 (1973) 3099. 